\title{
Lattice operators for scattering of particles with spin
}

\section{S. Prelovsek, ${ }^{a, b, c}$ U. Skerbis ${ }^{b}$ and C.B. Lang ${ }^{d}$}

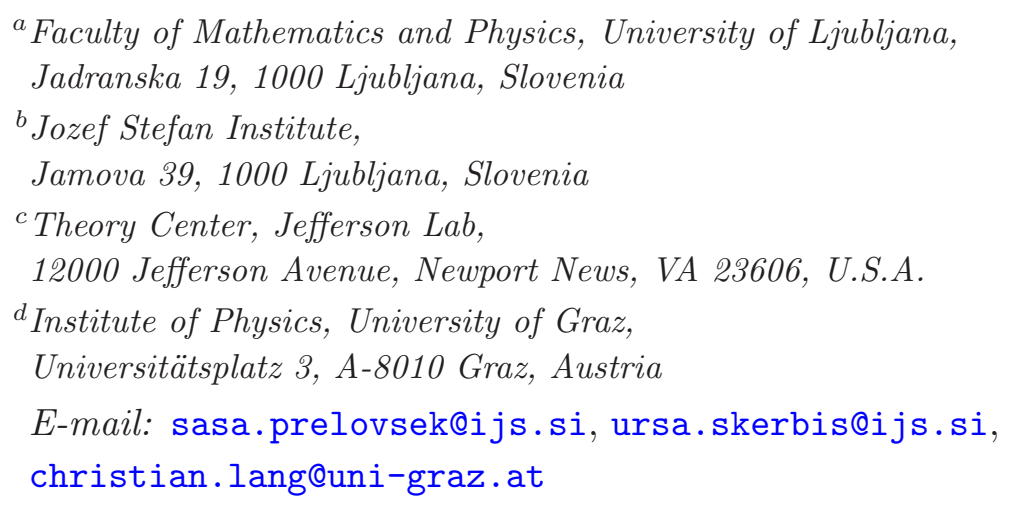

ABSTRACT: We construct operators for simulating the scattering of two hadrons with spin on the lattice. Three methods are shown to give the consistent operators for $P N, P V, V N$ and $N N$ scattering, where $P, V$ and $N$ denote pseudoscalar, vector and nucleon. Explicit expressions for operators are given for all irreducible representations at lowest two relative momenta. Each hadron has a good helicity in the first method. The hadrons are in a certain partial wave $L$ with total spin $S$ in the second method. These enable the physics interpretations of the operators obtained from the general projection method. The correct transformation properties of the operators in all three methods are proven. The total momentum of two hadrons is restricted to zero since parity is a good quantum number in this case.

KeYwords: Lattice QCD, Lattice Quantum Field Theory

ARXIV EPRINT: 1607.06738 


\section{Contents}

1 Introduction 1

2 Single-hadron operators and their transformations 2

3 Transformation properties of two-hadron operators 4

4 Two-hadron operators in three methods 5

4.1 Projection method 5

4.2 Helicity method 6

$\begin{array}{lll}\text { 4.2.1 } & \text { Construction of two-hadron helicity operators } & 7\end{array}$

$\begin{array}{lll}\text { 4.2.2 Subduction to irreducible representations } & 8\end{array}$

$\begin{array}{llr}4.3 & \text { Partial-wave method } & 9\end{array}$

$\begin{array}{lll}4.4 & \text { Relation between partial-wave and helicity operators } & 10\end{array}$

4.5 Method combining single particle irreducible representations 10

5 Operators with momenta $|p|=0,1 \quad 10$

$\begin{array}{lll}5.1 & P V \text { operators } & 11\end{array}$

$\begin{array}{lll}5.1 .1|p|=0 & 11\end{array}$

$\begin{array}{ll}5.1 .2|p|=1 & 11\end{array}$

$\begin{array}{lll}5.2 & P N, V N \text { and } N N \text { operators } & 13\end{array}$

6 Conclusions 13

$\begin{array}{ll}\text { A Proofs of transformation properties for operators } & 14\end{array}$

$\begin{array}{ll}\text { A.1 Projection method } & 14\end{array}$

$\begin{array}{lll}\text { A.2 Helicity method } & 14\end{array}$

$\begin{array}{lll}\text { A.3 Partial-wave method } & 15\end{array}$

B Technicalities $\quad \mathbf{1 6}$

B.1 Wigner D-matrices 16

$\begin{array}{lll}\text { B.2 Transformations of basis vectors and components } & 16\end{array}$

$\begin{array}{lll}\text { B.3 Two bases for vectors } & 17\end{array}$

$\begin{array}{lll}\text { B.4 Subduction matrices } \mathcal{S} & 17\end{array}$

$\begin{array}{ll}\text { C Expressions for two-hadron operators } & 17\end{array}$

$\begin{array}{ll}\text { C.1 PN operators } & 17\end{array}$

$\begin{array}{ll}\text { C.1.1 }|p|=0 & 17\end{array}$

$\begin{array}{ll}\text { C.1.2 }|p|=1 & 18\end{array}$

$\begin{array}{lll}\text { C.2 } & N V \text { operators } & 18\end{array}$

$\begin{array}{lll}\text { C.2.1 }|p|=0 & 18\end{array}$

$\begin{array}{ll}\text { C.2.2 }|p|=1 & 19\end{array}$ 


\section{Introduction}

The study of two-hadron interactions with lattice QCD requires operators that create and annihilate the two-hadron system with the desired quantum numbers. Systems of two hadrons with zero spin have been extensively studied and the corresponding scattering matrices for interactions of two pseudoscalars $(P)$ were extracted, for example $\pi \pi, K \pi$, .... The simulations of two-hadron systems where one or both hadrons carry non-zero spin are scarce, and those mostly focused on the partial-wave $L=0$. An example of a study that aimed also at higher partial waves considered nucleon-nucleon scattering [1].

There is a great need for lattice results on further channels or higher partial waves where one or both hadrons carry spin. We have in mind channels that involve the nucleon $N$ or/and vectors $V=J / \psi, \Upsilon_{b}, D^{*}, B^{*}, \ldots$ which are (almost) stable under strong interactions. The $P N$ or $V N$ scattering is crucial for ab-initio study of baryon resonances and pentaquark candidates, $P V$ is essential for mesonic resonances and tetraquark candidates, while $N N$ to grasp two-nucleon interaction and deuterium. ${ }^{1}$ We consider the most common simulations that are done in a box of size $L$ with periodic boundary conditions in space. The momenta $p$ of non-interacting single hadrons are multiples of $2 \pi / L$ in this case.

We focus on the system with total momentum zero where parity is a good quantum number. This has a practical advantage since fixing the parity gives a strong handle on which channels and partial waves can contribute as eigenstates in a given study. On the lattice the continuum symmetry is reduced to a discrete symmetry and one extracts the eigenstates that transform according to the chosen irreducible representation (irrep) $\Gamma$ of the discrete group $O_{h}^{(2)}$. Only the states with desired parity contribute to a given irrep when the total momentum is zero, while both even and odd partial waves contribute to a given irrep when the total momentum is not zero.

In this paper we present the general expressions to construct operators for scattering of particles with spin using three methods. The corresponding proofs of the correct transformation properties are given. Then the explicit expressions $H^{(1)}(p) H^{(2)}(-p)$ for $P V, P N$, $V N$ and $N N$ are presented for lowest two relative momenta $|p|=0,2 \pi / L$.

The reason to introduce two-hadron operators in a lattice simulation is to extract the eigenstates and their energies in a desired scattering channel. These energies render the scattering phase shift via the well-known Lüscher method [2], which was originally derived for the two spin-less particles. The relation has been generalized to the scattering of two

\footnotetext{
${ }^{1}$ The operators below can be straightforwardly generalized also to channels which involve scattering of particles with $J^{P}=0^{+}, 1^{+}, \frac{1}{2}^{-}$. Most of these hadrons decay strongly in Nature.
} 
particles with arbitrary spin [3-7] and the most general case is considered in [3]. This can be directly applied to extract the scattering matrix from the energies obtained using the operators presented in this work.

The three methods to construct operators complement each other and it is verified that all three methods lead to consistent results. The projection method is a general mathematical tool which leads to one or several operators $O_{\Gamma, r, n}$ that transform according to given irrep $\Gamma$ and row $r$. It is a "bottom-up" method, working entirely with lattice operators. But it gives little guidance on which continuum quantum numbers of total $J$, spin $S$, orbital momentum $L$ or single-particle helicities $\lambda_{1,2}$ will be related with a given operator. This is remedied with the helicity and partial-wave "top-down" methods where first the operators with good continuum quantum numbers $\left(J, P, \lambda_{1,2}\right)$ or $(J, L, S)$ are constructed and then subduced to the irreps $\Gamma$ of the discrete group. The hope is that the results indicate which linear combinations $O_{\Gamma, r, n}$ of various $n$ enhance couplings to the states with desired continuum quantum numbers. An analogous strategy for single-hadron states was proposed by the Hadron Spectrum Collaboration in [8]. We do not prove whether two particle operators also retain a "memory" of their construction origin. Future simulations analogous to the single-hadron case [8] will be necessary to explore how well that may be realized in practice.

Certain aspects of constructing the lattice operators for scattering of particles with spin were already presented before: partial-wave method in [1,9], projection method for example in [5], helicity states for single-hadrons in [10] and some considerations for two hadrons in $[9,11]$. The study [12] combines single particle irreps to two-particle operators. For this one needs the values of all corresponding Clebsch-Gordan coefficients to embed the product irreps of the single particle operators in the irreps of the two-particle frame (see section 4.5). Despite all previous work, various practical aspects and proofs are lacking to build a two-hadron operator related to desired continuum quantum numbers in a given irrep. Here we devise two-hadron helicity operators, verify their consistency with other two methods and provide related proofs. We also argue how simple non-canonical single-hadron operators can be used as building blocks for that purpose.

The paper is organized as follows. The single-hadron operators that are employed to build two-hadron operators are considered in section 2. The required transformations properties of two-hadron operators are given in section 3. The general expressions for two-hadron operators with three methods are presented in section 4, while the proofs of correct transformation properties are given in appendix A. The explicit operators for lowest two relative momenta are collected in sections 5 and $\mathrm{C}$. The necessary technicalities are delegated to appendix B.

\section{Single-hadron operators and their transformations}

The single-hadron operators $H(p)$ need to have certain transformation properties under rotations $R$ and inversion $I$ in order to build two-hadron operators $H^{(1)}(p) H^{(2)}(-p)$ with desired transformation properties. The states and creation operators $H_{m_{s}}^{\dagger}(p)$ with spin $s$ 
transform as

$$
\begin{array}{rlrl}
R\left|p, s, m_{s}\right\rangle & =\sum_{m_{s}^{\prime}} D_{m_{s}^{\prime} m_{s}}^{s}(R)\left|R p, s, m_{s}^{\prime}\right\rangle, & & I\left|p, s, m_{s}\right\rangle=(-1)^{P}\left|-p, s, m_{s}\right\rangle \\
R H_{m_{s}}^{\dagger}(p) R^{-1} & =\sum_{m_{s}^{\prime}} D_{m_{s}^{\prime} m_{s}}^{s}(R) H_{m_{s}^{\prime}}^{\dagger}(R p), & I H_{m_{s}}^{\dagger}(p) I=(-1)^{P} H_{m_{s}}^{\dagger}(-p) .
\end{array}
$$

For a particle at rest $m_{s}$ is a good quantum number of the spin-component $S_{z}$. The $m_{s}$ is generally not a good quantum number for $H_{m_{s}}(p \neq 0)$; in this case it denotes the eigenvalue of $S_{z}$ for the corresponding field $H_{m_{s}}(0)$, which has good $m_{s}$. The transformations of annihilation operators $H_{m_{s}}(p)$ are obtained by the hermitian conjugation

$$
R H_{m_{s}}(p) R^{-1}=\sum_{m_{s}^{\prime}} D_{m_{s} m_{s}^{\prime}}^{s}\left(R^{-1}\right) H_{m_{s}^{\prime}}(R p), \quad I H_{m_{s}}(p) I=(-1)^{P} H_{m_{s}}(-p),
$$

where the following properties of the Wigner D-matrix and $\mathrm{R}$ are used throughout this work

$$
D(R)=D^{\dagger}\left(R^{\dagger}\right), \quad R^{\dagger}=R^{-1}, \quad D\left(R_{1} R_{2}\right)=D\left(R_{1}\right) D\left(R_{2}\right) .
$$

We employ the conventional definition of $D$ used in $[13,14]$ and discussed in appendix B.1. Note that the $z$-component of spin $m_{s}$ is a good quantum number for a particle at rest.

The pseudoscalar fields $P\left(J^{P}=0^{-}\right)$, vector fields $V\left(J^{P}=1^{-}\right)$and nucleon fields $N$ $\left(J^{P}=\frac{1}{2}^{+}\right)$are considered, together with their mutual two-hadron scattering channels later on. One can choose the simplest annihilation operators that transform according to (2.2), for example

$$
\begin{aligned}
P(p) & =\sum_{x} \bar{q}(x) \gamma_{5} q(x) e^{i p x} \\
V_{m_{s}= \pm 1}(p) & =\frac{\mp V_{x}(p)+i V_{y}(p)}{\sqrt{2}}, \quad V_{m_{s}=0}(p)=V_{z}(p), \quad V_{i}(p)=\sum_{x} \bar{q}(x) \gamma_{i} q(x) e^{i p x}, i=x, y, z \\
N_{m_{s}=1 / 2}(p) & =\mathcal{N}_{\mu=1}(p), \mathcal{N}_{m_{s}=-1 / 2}(p)=\mathcal{N}_{\mu=2}(p), \quad \mathcal{N}_{\mu}(p)=\sum_{x} \epsilon_{a b c}\left[q^{a T}(x) C \gamma_{5} q^{b}(x)\right] q_{\mu}^{c}(x) e^{i p x},
\end{aligned}
$$

where $p$ and $x$ are three-vectors and the time index is omitted. The $\mathcal{N}_{1,2}$ are the upper two components of Dirac four-spinor $\mathcal{N}_{\mu=1, \ldots, 4}$ in the Dirac basis. The relations for the vector states $\left|V_{m_{s}=1}\right\rangle=\left(-\left|V_{x}\right\rangle-i\left|V_{y}\right\rangle\right) / \sqrt{2}$ versus annihilation operators $V_{m_{s}=1}=\left(-V_{x}+i V_{y}\right) / \sqrt{2}$ are discussed in appendix B.3. The operators $P, N_{1 / 2,-1 / 2}$ and $V_{i=x, y, z}$ like (2.4) will be basic building blocks of our two-hadron operators.

Since all two-hadron operators will entail transformations of annihilation fields $H=$ $P, V, N$, we present them explicitly

$$
\begin{aligned}
R P(p) R^{-1} & =P(R p), \quad I P(p) I=-P(-p) \\
R V_{i}(p) R^{-1} & =T_{j i}^{s=1}(R)^{*} V_{j}(R p)=\exp (-i \vec{n} \vec{J} \omega)_{j i} V_{j}(R p), \quad I V_{i}(p) I=-V_{i}(-p) \quad i, j=x, y, z \\
R N_{m_{s}}(p) R^{-1} & =D_{m_{s}^{\prime} m_{s}}^{s}(R)^{*} N_{m_{s}^{\prime}}(R p)=\left[\exp \left(-\frac{i}{2} \vec{n} \vec{\sigma} \omega\right)\right]_{m_{s}^{\prime} m_{s}}^{*} N_{m_{s}^{\prime}}(R p), \quad I N_{m_{s}}(p) I=N_{m_{s}}(-p)
\end{aligned}
$$

with $m_{s, s^{\prime}}= \pm 1 / 2$ and $\left(J_{k}\right)_{i j}=-i \epsilon_{i j k}$ rendering real $T^{s=1}$. The fields transform as basisvectors, which is contrasted to the transformation of components in appendix B.2. The 
transformations of $V_{i}(p)$ in (2.5) can be used to verify the transformations (2.2) of the fields $V_{m_{s}}(p)$ defined in (2.4).

Note that canonical hadron fields $H_{m_{s}}^{(c)}(p) \equiv L(p) H_{m_{s}}(0)$ also transform under rotations as given in $(2.2),{ }^{2}$ but those would be less practical to implement. The canonical fields are obtained from the rest fields $H_{m_{s}}(0)$ (which have good $m_{s}$ ) with the boost $L(p)$ from 0 to $p$. The boost of $V_{m_{s}=1}(0)$ to $p_{x} \propto e_{x}$ would render $V_{m_{s}=1}^{(c)}\left(p_{x}\right)=\left[-\gamma V_{x}\left(p_{x}\right)+i V_{y}\left(p_{x}\right)\right] / \sqrt{2}$ which depends on $\gamma=\left(1-v^{2}\right)^{1 / 2}$. The boost of $N_{1 / 2}(0)=\mathcal{N}_{1}(0)$ to $p_{x}$ would render $N_{1 / 2}^{(c)}\left(p_{x}\right) \propto \mathcal{N}_{1}(0)+\frac{p_{x}}{m+E} \mathcal{N}_{4}$, which contains also lower components and depends on the mass $m$. We will employ the simpler fields $V$ and $N(2.4)$ that have all the required transformation properties (2.2), (2.5) to prove the correct transformations of the twohadron operators in appendix A. The fields (2.4) agree with the canonical fields for $p=0$.

\section{Transformation properties of two-hadron operators}

We consider only two-hadron states with total momentum zero $P_{\text {tot }}=0$ that have good parity $P$. These also have good total spin $J$ and its $z$-component $m_{J}$ in continuum, where the annihilation operators have to transform as (2.2)

$$
R O^{J, m_{J}}\left(P_{\mathrm{tot}}=0\right) R^{-1}=\sum_{m_{J}^{\prime}} D_{m_{J} m_{J}^{\prime}}^{J}\left(R^{-1}\right) O^{J, m_{J}^{\prime}}(0) R \in O^{(2)}, I O^{J, m_{J}}(0) I=(-1)^{P} O^{J, m_{J}}(0) .
$$

Such continuum-like operators will present an intermediate step below and the only relevant rotations will be those contained in the discrete group.

On the cubic lattice the continuum rotation group is reduced to the cubic group $R \in O$ with 24 elements for integer $J$. These are given in terms of the rotation angle $-\pi \leq \omega<\pi$ around vector $\vec{n}$ in positive direction and we use the ordering $i=1, \ldots, 24$ listed in table A.1 of [16]. The double-cover group $O^{2}$ has the same 24 elements, and additional 24 elements $(i=25, \ldots 48)^{3}$ with the same $\vec{n}$ and angle $\pi \leq \omega<3 \pi$ where $\omega_{i+24}=\omega_{i}+2 \pi$. The number of symmetry elements gets doubled to $\tilde{R}=\{R, I R\} \in O_{h}^{(2)}$ when inversion is a group element.

The representations (3.1) with given $J$ and $m_{J}$ are reducible under $O_{h}^{(2)}$, so we seek the states/operators that transform according to the corresponding irreducible representation (irrep) $\Gamma$ and row $r$

$$
\begin{array}{rlrl}
R|\Gamma, r\rangle & =\sum_{r^{\prime}} T_{r^{\prime}, r}^{\Gamma}(R)\left|\Gamma, r^{\prime}\right\rangle & R \in O^{(2)}, & I|\Gamma, r\rangle=(-1)^{P}|\Gamma, r\rangle, \\
R O_{\Gamma, r} R^{-1}=\sum_{r^{\prime}} T_{r, r^{\prime}}^{\Gamma}\left(R^{-1}\right) O_{\Gamma, r^{\prime}} & R \in O^{(2)}, & I O_{\Gamma, r} I=(-1)^{P} O_{\Gamma, r} .
\end{array}
$$

The systems with integer $J$ transform according to irreps $\Gamma=A_{1,2}^{ \pm}, E^{ \pm}, T_{1,2}^{ \pm}$, while systems with half-integer $J$ according to $\Gamma=G_{1,2}^{ \pm}, H^{ \pm}$as summarized in table 1 . The upper index stands for parity and the number of rows $r=1, \ldots, \operatorname{dim}_{\Gamma}$ is equal to the dimensionality of irrep $\Gamma$. We employ the same conventions for rows in all irreps as in [16]. ${ }^{4}$ The explicit representations $T_{r, r^{\prime}}^{\Gamma}(R)$ for $R \in O^{(2)}$ are given in appendix A of [16]. ${ }^{5}$

\footnotetext{
${ }^{2}$ See for example eq. (2.5.23) of Weinberg [15] with $W(\mathcal{R}, p)=\mathcal{R}$.

${ }^{3}$ Our ordering and range of $\omega$ for $O^{2}$ differs from table A.3 in [16].

${ }^{4}$ Those are partly discussed in our appendix B.4.

${ }^{5}$ In [16] the $T_{r^{\prime}, r}^{\Gamma}$ are denoted by $\left(R_{i}\right)_{\alpha \beta}$.
} 


\begin{tabular}{|c|c|}
\hline $\mathrm{J}$ & $\Gamma\left(\operatorname{dim}_{\Gamma}\right)$ \\
\hline 0 & $A_{1}(1)$ \\
$\frac{1}{2}$ & $G_{1}(2)$ \\
1 & $T_{1}(3)$ \\
$\frac{3}{2}$ & $H(4)$ \\
2 & $E(2) \oplus T_{2}(3)$ \\
$\frac{5}{2}$ & $H(4) \oplus G_{2}(2)$ \\
3 & $A_{2}(1) \oplus T_{1}(3) \oplus T_{2}(3)$ \\
\hline
\end{tabular}

Table 1. Continuum spins $J$, corresponding lattice irreps $\Gamma$ and their dimension $\operatorname{dim}_{\Gamma}$ for group $O^{(2)}$.

As indicated above, here we consider only two-hadron states with total momentum zero. Both even and odd partial waves contribute to a given irrep when total momentum is not zero. Working with operators that mix parity is no problem in principle and has already been considered for, e.g., pseudoscalar-pseudoscalar scattering. It leads to more eigenstates in the given irreducible representation and one has to apply further assumptions and tools to extract the scattering phase shift. Note that in both cases, exact or mixed parity, the lattice irreps may still couple to a tower of higher partial waves. The phase shifts for $\ell \leq 2$ in the parity mixed case have been successfully extracted, for example, via the parameterization of the S-matrix by the Hadron Spectrum Collaboration in $[17,18]$.

\section{Two-hadron operators in three methods}

Here we present two-hadron operators with total momentum zero that transform according to (3.2). They will be constructed by three methods, where continuum-like operators (3.1) will appear as an intermediate step in two of the methods. Their correct transformation properties (3.1), (3.2) are proven in appendix A.

\subsection{Projection method}

This is a well-known mathematical method, where a projector to the desired irrep $\Gamma$ and row $r$ is used on an arbitrary operator (see for example section 4.19 of [19])

$$
\begin{aligned}
\| p|, \Gamma, r, n\rangle & =\sum_{\tilde{R} \in O_{h}^{(2)}} T_{r, r}^{\Gamma}(\tilde{R})^{*} \tilde{R}\left[\left|2,-p, s_{2}\right\rangle^{a}\left|1, p, s_{1}\right\rangle^{a}\right] \\
O_{|p|, \Gamma, r, n} & =\sum_{\tilde{R} \in O_{h}^{(2)}} T_{r, r}^{\Gamma}(\tilde{R}) \tilde{R} H^{(1), a}(p) H^{(2), a}(-p) \tilde{R}^{-1}, \quad n=1, \ldots, n_{\max } .
\end{aligned}
$$

The $H^{a}$ are arbitrary single hadron lattice operators of desired $|p|$ and any $p$ and $m_{s}$, for example operators (2.4) or their linear combinations; the other index (1) and (2) denotes the particle type. We have taken $H^{a}$ with all possible combinations of direction $p$ and polarizations of both particles $m_{s 1}$ and $m_{s 2}$ (for vectors we chose $V_{x}, V_{y}$ and $V_{z}$ as $H^{a}$ ). 
For fixed $|p|, \Gamma$ and $r$ one can get one or more linearly independent operators $O_{|p|, \Gamma, r, n}$ which are indicated by $n$. As an illustration we present two-linearly independent $P V$ operators

$$
O_{|p|=1, T_{1}^{+}, r=3, n=1} \propto \sum_{p= \pm e_{z}} \mathrm{P}(p) V_{z}(-p), \quad O_{|p|=1, T_{1}^{+}, r=3, n=2} \propto \sum_{p= \pm e_{x}, \pm e_{y}} \mathrm{P}(p) V_{z}(-p), \quad n_{\max }=2,
$$

while others are listed in the section 5 and appendix C.

The $\tilde{R} \in O_{h}^{(2)}$ in (4.1) is the operator symbolising rotations $R$ possibly combined with inversions ( $R$ is reserved in this article for rotations only) and one uses in practice

$$
\tilde{R} H^{(1)} H^{(2)} \tilde{R}^{-1}=\tilde{R} H^{(1)} \tilde{R}^{-1} \tilde{R} H^{(2)} \tilde{R}^{-1}, \quad \tilde{R} \in O_{h}^{(2)}
$$

where the action of rotation $R$ or inversion $I$ on all $H=P, V, N$ are given in $(2.2,2.5)$. The representation matrices $T^{\Gamma}(\tilde{R})$ for all elements $\tilde{R}$ are listed for all irreps in appendix A of [16]. The proof of correct transformation is shown for completeness in appendix A.

The projection method is very general, but it does not offer physics intuition what $O_{|p|, \Gamma, r, n}$ with different $n$ represent in terms of the continuum quantum numbers. This will be remedied with the next two methods, that indicate which linear combinations of $O_{n}$ correspond in the continuum to certain partial waves or helicity quantum numbers.

\subsection{Helicity method}

The states with good helicity in the continuum scattering have been thoroughly discussed by Jacob and Wick [13]. They have been considered for one-hadron states on the lattice by the Hadron-Spectrum collaboration [10], but they have not been widely used for two-hadron scattering on the lattice yet.

The $m_{s}$ is a good quantum number for particles with $p=0$ or $p_{z} \propto e_{z}$ since $L_{z}=0$ and $S_{z}=J_{z}$, while it is generally not a good quantum number for arbitrary $p \neq 0$. Here $L_{z}, S_{z}$ and $J_{z}$ denote components of the orbital, spin and total angular momentum, respectively. The advantage of the helicity $h$

$$
h \equiv \frac{S \cdot p}{|p|}
$$

is that it is a good quantum number for arbitrary $p$, where $S$ and $p$ are three-vectors. To obtain a helicity state, one starts from a state with momentum $p_{z} \propto e_{z}$ and $\left|p_{z}\right|=|p|$ that has good $m_{s}$. This state is rotated from $p_{z}$ to desired direction of $p$ with $R_{0}^{p}$,

$$
|p, s, \lambda\rangle^{h} \equiv R_{0}^{p}\left|p_{z}, s, m_{s}\right\rangle, \quad H_{\lambda}^{h}(p) \equiv R_{0}^{p} H_{m_{s}=\lambda}\left(p_{z}\right)\left(R_{0}^{p}\right)^{-1}, \quad p_{z} \propto e_{z}, \quad\left|p_{z}\right|=p .
$$

The upper index $h$ indicates that the polarisation index $(\lambda)$ stands for the helicity of the particle (not $m_{s}$ ). Different choices of $R_{0}^{p}$ are possible, which lead to different phases in the definition of $|p, s, \lambda\rangle^{h}$. We do not restrict to a specific choice of $R_{0}^{p}$ since our resulting two-particle operator will be independent of this (except for the overall phase of operator, which is irrelevant). Simple examples of $H_{p_{z}, m_{s}=\lambda}$ can be read-off from (2.4) and the action of $R_{0}^{p}$ on them is given by $(2.5)$. 
The arbitrary rotation $R$ rotates the momentum $p$ and the spin $S$ in the same way, so the helicity $h \propto S \cdot p$ of the state remains the same and only the direction of the momentum changes $[10,13]$

$$
R|p, s, \lambda\rangle^{h}=e^{-i \varphi(R)}|R p, s, \lambda\rangle^{h}, \quad R H_{\lambda}^{h}(p) R^{-1}=e^{i \varphi(R)} H_{\lambda}^{h}(R p),
$$

where only the phase $\varphi(R)$ depends on $R$.

\subsubsection{Construction of two-hadron helicity operators}

The starting point is a two-particle state composed of $\left|p, s_{1}, \lambda_{1}\right\rangle^{h}$ and $\left|-p, s_{2}, \lambda_{2}\right\rangle^{h}$, where $p$ can be chosen as arbitrary momentum in a given shell $|p|$, and $\lambda_{1,2}$ are chosen helicities. The two-particle helicity state and the annihilation operator with the correct transformation properties under rotation (3.1) are

$$
\begin{aligned}
\| p\left|, J, m_{J}, \lambda_{1}, \lambda_{2}, \lambda\right\rangle^{h} & =\sum_{R \in O^{(2)}} D_{\lambda, m_{J}}^{J}\left(R^{-1}\right) R\left[\left|p, s_{1}, \lambda_{1}\right\rangle^{h}\left|-p, s_{2}, \lambda_{2}\right\rangle^{h}\right] \\
O^{|p|, J, m_{J}, \lambda_{1}, \lambda_{2}, \lambda} & =\sum_{R \in O^{(2)}} D_{m_{J}, \lambda}^{J}(R) R H_{\lambda_{1}}^{(1), h}(p) H_{\lambda_{2}}^{(2), h}(-p) R^{-1}
\end{aligned}
$$

with the proof for operators given in appendix A. Note that the labels on the left-hand side should just indicate the origin of construction; they would correspond to the continuum quantum numbers if $R$ was summed over the continuum rotation group. The main difference with respect to the continuum case [13] is the discrete sum over the elements of the discrete group $R \in O$ for integer $J$ and $R \in O^{2}$ for half-integer $J$. Appendix B.1 provides $D$-matrices ${ }^{6}$ for $R$ in $O$ and double-cover $O^{2}$. The helicity of the two-particle state is $\lambda=\lambda_{1}-\lambda_{2}$ for all cases we consider. ${ }^{7}$

Note that the operator (4.6) is a sum of states with fixed $\lambda_{1,2}$ since the helicity of each particle is conserved with rotation (4.5),

$$
O^{|p|, J, m_{J}, \lambda_{1}, \lambda_{2}, \lambda}=\sum_{R \in O^{(2)}} D_{m_{J}, \lambda}^{J}(R) e^{i \Phi(R)} H_{\lambda_{1}}^{(1), h}(R p) H_{\lambda_{2}}^{(2), h}(-R p) .
$$

The phase $\Phi(R)$ depends also on the choice of $R_{0}$ through the definition of the helicity state (4.4). We do not explicitly determine $\Phi(R)$, but rather directly employ expressions (4.6) and (4.8) to get explicit results for the interpolators in section 5.

The final helicity operator with desired parity $P= \pm 1$ is obtained from (4.6) by parity projection $\frac{1}{2}(\mathcal{O}+P I \mathcal{O} I)$

$$
\begin{aligned}
O^{|p|, J, m_{J}, P, \lambda_{1}, \lambda_{2}, \lambda}=\frac{1}{2} \sum_{R \in O^{(2)}} D_{m_{J}, \lambda}^{J}(R) & R R_{0}^{p}\left[H_{m_{s_{1}}=\lambda_{1}}^{(1)}\left(p_{z}\right) H_{m_{s_{2}}=-\lambda_{2}}^{(2)}\left(-p_{z}\right)\right. \\
& \left.+P I H_{m_{s_{1}}=\lambda_{1}}^{(1)}\left(p_{z}\right) H_{m_{s_{2}}=-\lambda_{2}}^{(2)}\left(-p_{z}\right) I\right]\left(R_{0}^{p}\right)^{-1} R^{-1},
\end{aligned}
$$

\footnotetext{
${ }^{6}$ One has to use the same rotation in $D$ and in the transformation of fields. The additional rotation of $2 \pi$ that is present in half of the $O^{2}$ elements renders factors $(-1)^{J}$ from $D^{J}(R)$ and $(-1)^{s_{1}}(-1)^{s_{2}}$ from the transformations of the both fields in (4.6).

${ }^{7}$ In the continuum this follows from the continuum integration over Euler angle $\gamma[13]$.
} 
where we have expressed $H^{h}$ (4.4) with fields $H_{m_{s}}\left(p_{z}\right)(2.4)$ that have good $m_{s}$. The actions of inversion $I$ and the rotation $R$ on the fields $H_{m_{s}}$ are given in (2.5). One chooses particular $p$ with given $|p|$ and performs rotation $R_{0}^{p}$ from $p_{z}$ to $p$. There are several possible choices of $R_{0}^{p}$, but they lead only to different overall phases for the whole operator (4.8), which is irrelevant. ${ }^{8}$

The operators for $|p|=1$ (in units of $2 \pi / L$ ) will be explicitly presented in section 5 . We take the simplest choice $p=p_{z}=(0,0,1)$ and $R_{0}^{p}=\mathbf{1}$ in $(4.8)^{9}$

$$
\begin{aligned}
O^{|p|=1, J, m_{J}, P, \lambda_{1}, \lambda_{2}, \lambda}=\frac{1}{2} \sum_{R \in O^{(2)}} D_{m_{J}, \lambda}^{J}(R) R[ & H_{m_{s 1}=\lambda_{1}}^{(1)}\left(p_{z}\right) H_{m_{s_{2}}=-\lambda_{2}}^{(2)}\left(-p_{z}\right) \\
& \left.+P I H_{m_{s 1}=\lambda_{1}}^{(1)}\left(p_{z}\right) H_{m_{s_{2}}=-\lambda_{2}}^{(2)}\left(-p_{z}\right) I\right] R^{-1} .
\end{aligned}
$$

As an illustration we present two $P V$ operators with the same $J^{P}$,

$$
\begin{aligned}
& O^{|p|=1, J=1, m_{J}=0, P=+, \lambda_{V}=0} \propto \sum_{p= \pm e_{z}} \mathrm{P}(p) V_{z}(-p), \\
& O^{|p|=1, J=1, m_{J}=0, P=+, \lambda_{V}=1} \propto \sum_{p= \pm e_{x}, \pm e_{y}} \mathrm{P}(p) V_{z}(-p) .
\end{aligned}
$$

\subsubsection{Subduction to irreducible representations}

The operators (4.8) would correspond to the irreducible representations only for the continuum rotation group $R \in \mathrm{SO}(3)$ in which case $J$ indicates the continuum quantum number. These operators (4.8) represent reducible representation under the reduced discrete group $O^{(2)}$. In simulations one needs to employ operators, which transform according to irreducible representations $\Gamma$ and row $r$ of $G=O^{(2)}$. Those are obtained from the continuum-like operators $O^{J, m_{J}}$ by the subduction $[8,20]$

$$
O_{|p|, \Gamma, r}^{\left[J, P, \lambda_{1}, \lambda_{2}, \lambda\right]}=\sum_{m_{J}} \mathcal{S}_{\Gamma, r}^{J, m_{J}} O^{|p|, J, m_{J}, P, \lambda_{1}, \lambda_{2}, \lambda} .
$$

The subduction coefficients $\mathcal{S}$ are real and are given in appendices of [8] and [20] for all irreps. For the $T_{1,2}$ irreps our convention for rows is different and the corresponding coefficients $\mathcal{S}$ are listed in appendix B.4.

This strategy, to start from operators with continuum spin $O^{J, m_{J}}$ and subduce them to irreps $O_{\Gamma, r}^{[J]}$, was proposed by the Hadron Spectrum Collaboration in [8]. It was then extensively employed for single hadrons by the same collaboration. ${ }^{10}$ The subduced operators $O_{\Gamma, r}^{[J]}$ were found to "carry the memory" of the spin $J$, where they were subduced from and dominantly couple to the lattice eigenstates with this spin. Whether that expectation holds for two-particle operators combining spin and angular momentum is unclear; if the behaviour is like for the single-particle operators one may expect that the subduced operators $O_{|p|, \Gamma, r}^{\left[J, P, \lambda_{1}, \lambda_{2}, \lambda\right]}$ will dominantly represent eigenstates with continuum quantum numbers $J, \lambda_{1}, \lambda_{2}, \lambda$.

\footnotetext{
${ }^{8}$ The $p$ is the same for all terms (4.8), so the phase related to the choices of $R_{0}^{p}$ is the same in all terms.

${ }^{9} \mathrm{Such}$ a choice is not available for some shells, for example $|p|^{2}=2$. In this case one chooses some $p$ available in the shell and evaluates (4.8).

${ }^{10}$ This collaboration employed operators for two spin-less particles, which were obtained by combining $O_{\Gamma_{1}, r_{1}} \times O_{\Gamma_{2}, r_{2}} \rightarrow O_{\Gamma, r}$ using corresponding Clebsch-Gordan coefficients [11].
} 


\subsection{Partial-wave method}

Often one is interested in the scattering of two hadrons in a given partial wave $L$. The orbital angular momentum $L$ and total spin $S$ are not separately conserved, so several $(L, S)$ combinations can render the same $J, m_{J}$ and $P$, which are good quantum numbers. Nevertheless, the $L$ and $S$ are valuable physics quantities to label continuum annihilation field operator

$$
O^{|p|, J, m_{J}, L, S}=\sum_{m_{L}, m_{S}, m_{s 1}, m_{s 2}} C_{L m_{L}, S m_{S}}^{J m_{J}} C_{s_{1} m_{s 1}, s_{2} m_{s 2}}^{S m_{S}} \sum_{R \in O} Y_{L m_{L}}^{*}(\widehat{R p}) H_{m_{s 1}}^{(1)}(R p) H_{m_{s 2}}^{(2)}(-R p)
$$

The operator has parity $P=P_{1} P_{2}(-1)^{L}$ and its correct transformation property under rotation (3.1) is demonstrated in appendix A. The operator was considered for nucleonnucleon scattering already in [1], where the proof was not presented (this reference uses $Y_{L m_{L}}$ where we have $Y_{L m_{L}}^{*}$ ). The $C$ are Clebsch-Gordan coefficients, $p$ is an arbitrary momentum with desired $|p|, Y_{L m_{L}}(\widehat{R p})$ is a spherical harmonic for direction $(\theta, \phi)$ given by the normalized vector $\widehat{R p}$. Simple choices of one-particle annihilation operators $H$ are listed in (2.4). The sum is over $R \in O$ for integer as well as half-integer $J$, since rotations for angle $\omega$ and $\omega+2 \pi$ have the same effect on $p$. Here is an example of two $P V$ operators with the same $J$ and $S$

$$
\begin{aligned}
O^{|p|=1, J=1, m_{J}=0, L=0, S=1} & \propto \sum_{p= \pm e_{x}, \pm e_{y}, \pm e_{z}} \mathrm{P}(p) V_{z}(-p) \\
O^{|p|=1, J=1, m_{J}=0, L=2, S=1} & \propto \sum_{p= \pm e_{x}, \pm e_{y}} \mathrm{P}(p) V_{z}(-p)-2 \sum_{p= \pm e_{z}} \mathrm{P}(p) V_{z}(-p) .
\end{aligned}
$$

The operators (4.11) that are labeled by the continuum $J$ are reducible under reduced group $O^{(2)}$, as discussed in section 4.2.2. The operators that transform according to irrep $\Gamma$ and row $r$ of $G=O^{(2)}$ are obtained from $O^{J, m_{J}}$ by the subduction [8]

$$
O_{|p|, \Gamma, r}^{[J, L, S]}=\sum_{m_{J}} \mathcal{S}_{\Gamma, r}^{J, m_{J}} O^{|p|, J, m_{J}, L, S}
$$

where $\mathcal{S}$ are the same subduction coefficients as in (4.10). One expects that the subduced operators $O_{|p|, \Gamma, r}^{[J, L, S]}$ carry the memory of continuum $J, L, S$ and dominantly couple to eigenstates with these quantum numbers. This depends on how close one is to the physical limit and whether there may be an actual physical transition ${ }^{11}$ between states of different $(L, S)$ in the given chanel $J^{P}$. If this transition is small, one can hope that operators (4.12) would be valuable handles for certain lower values of $J$ and $L$, which are of most practical importance. Certain higher $L^{\prime}>L$ inevitably lead to the same operators (or their linear combinations), and $O_{\Gamma}^{[J, L, S]}$ could provide an effective handle on the low partial wave $L$ particularly if the corresponding higher partial waves $L^{\prime}$ are negligible (as illustrated in section 5.1.2).

\footnotetext{
${ }^{11}$ For example $L=0$ and $L=2$ mix in continuum in the $P V$ and $N N$ channel with $S=1$ and $J^{P}=1^{+}$.
} 


\subsection{Relation between partial-wave and helicity operators}

Operators calculated in partial-wave method (4.11) are linear combinations of operators calculated by helicity method (4.8)

$$
O^{|p|, J, m_{J}, S, L}=\sqrt{\frac{2 L+1}{4 \pi}} \sum_{\lambda=-S}^{S} \sum_{\lambda_{1}, \lambda_{2}} \sum_{\lambda^{\prime}} D_{\lambda^{\prime}, \lambda}^{J}\left(R_{0}^{p}\right) C_{L 0, S \lambda}^{J \lambda} C_{s_{1} \lambda_{1}, s_{2}-\lambda_{2}}^{S \lambda} O^{|p|, J, m_{J}, \lambda^{\prime}, \lambda_{1}, \lambda_{2}}
$$

where the proof of the relation is presented in the appendix D. For $|p|=1$, we take the reference momentum $p=R_{0}^{p} p_{z}$ along the z-direction, so $R_{0}^{p}$ and $D^{J}\left(R_{0}^{p}\right)$ are identity matrices.

\subsection{Method combining single particle irreducible representations}

Let us mention also a fourth method, which is not applied here. This approach [12] combines single particle irreps $\Gamma_{1,2}$ (for the cubic group at $p=0$ or for the little groups at $p \neq 0)$ to two-particle operators in irrep $\Gamma: \Gamma_{1} \otimes \Gamma_{2} \rightarrow \Gamma$. One hopes that this way one may profit from the optimized single particle operators for given $\Gamma_{1,2}$, as for example in the $P P$ study [17]. One needs the values of all corresponding Clebsch-Gordan coefficients to embed the product irreps of the single particle operators in the irreps of the two-particle frame. The method has been, for example, used in PP scattering [17] where the Clebsch-Gordan coefficients are known [11]. The reference [12] provides which Clebsch-Gordan coefficients are non-zero for scattering of particles with spin. This approach does not relate the resulting operators to continuum quantum numbers like $L$ or $S$. Nevertheless, it might turn out to be particularly valuable for constructing two-hadron operators with non-zero total momenta.

\section{Operators with momenta $|p|=0,1$}

The explicit expressions for annihilation operators $H^{(1)}(p) H^{(2)}(-p)$ for $P V, P N, V N$ and $N N$ scattering in three methods are collected here and in appendix C. We restrict to lowest two momenta $|p|=0,1$ (in units of $2 \pi / L$ ) for the individual hadrons on the lattice of size $L$ with periodic boundary conditions in space. Operators from the projection method $O_{|p|, \Gamma, r, n}$ will be given first, where $n=1, \ldots, n_{\max }$ indicate all linearly independent combinations for given $\Gamma$ and $r$. Then operators from helicity method $O_{|p|, \Gamma, r}^{\left[J, P, \lambda_{1}, \lambda_{2}, \lambda\right]}$ and partial-wave method $O_{|p|, \Gamma, r}^{[J, L, S]}$ will be expressed as linear combinations of known operators $O_{|p|, \Gamma, r, n}$ obtained by the projection method. This indicates which linear combinations have to be employed in a simulation to enhance a coupling to the state with continuum quantum numbers $J, P, \lambda_{1}, \lambda_{2}$ or $J, L, S$. This coupling is expected to be favourable when the studied system is approaching the physical limit, if certain higher partial waves are suppressed and if the physical mixing of different $(L, S)$ in given $J^{P}$ channel is small.

In both, helicity and partial wave, methods we consider $J \leq 2$ for integer and $J \leq 5 / 2$ for half-integer $J$. The helicity operator will not be given for $|p|=0$ as helicity $h$ (4.3) is intended for non-zero momentum. This operator satisfies

$$
O_{\Gamma, r}^{\left[J, P, \lambda_{1}, \lambda_{2}, \lambda=\lambda_{1}-\lambda_{2}\right]} \propto O_{\Gamma, r}^{\left[J, P,-\lambda_{1},-\lambda_{2},-\lambda\right]}
$$


since $h$ (4.3) reverses sign if $p \rightarrow-p$ and $S \rightarrow S$, while $p \rightarrow-p$ only multiplies the operator by \pm 1 depending on parity. The $m_{J}$ will appear as an index in helicity and partialwave operators in cases when only one $m_{J}$ is present in subduction to given irrep and row (4.10), (4.12). The vector fields are expressed in the $V_{x, y, z}$ basis (2.4), which are most straightforward for implementation. The normalization of each operator is arbitrary. For brevity, only the first row $r=1$ is displayed, which should suffice for an actual simulation. Other rows and $|p|$ can be obtained from the general expressions for $O$ above.

We verified that all partial-wave and helicity operators are related by (4.13). Also, we confirmed that the number of linearly independent operators in each irrep agrees with the fourth method [12] in section 4.5, as elaborated for the $P V$ case below. The number of operators based in this method can be deduced based on table I (for $p=0$ ) and table III (for $|p|=1$ ) of [12].

Before listing the operators, let us point out again the key expressions that have been used to derive them. The operators from projection method are obtained using (4.1) with representations $T^{\Gamma}(\tilde{R})$ listed in appendix A of [16]. The helicity operator (4.9) for $|p|=1$ is subduced to irreps by (4.10), while the partial-wave operator (4.11) comes from the subduction with (4.12). The simple examples of the fields $H_{m_{s}}$ are collected in (2.4) with their transformations in (2.2), (2.5) and Wigner-D functions in (B.1).

\section{1 $P V$ operators}

\subsection{1 $|p|=0$}

$$
\begin{aligned}
& T_{1}^{+}: \\
& O_{T_{1}^{+}, r=1}=\mathrm{P}(0) V_{x}(0) \\
& O_{T_{1}^{+}, r=1}^{[J=1, L=0, S=1]}=O_{T_{1}^{+}, r=1}
\end{aligned}
$$

Operators for other irreps are equal to 0.

5.1.2 $|p|=1$

$$
\begin{aligned}
& A_{1}^{-}: \\
& O_{A_{1}^{-}, r=1}=\mathrm{P}\left(e_{x}\right) V_{x}\left(-e_{x}\right)-\mathrm{P}\left(-e_{x}\right) V_{x}\left(e_{x}\right)+\mathrm{P}\left(e_{y}\right) V_{y}\left(-e_{y}\right)-\mathrm{P}\left(-e_{y}\right) V_{y}\left(e_{y}\right) \\
& \quad+\mathrm{P}\left(e_{z}\right) V_{z}\left(-e_{z}\right)-\mathrm{P}\left(-e_{z}\right) V_{z}\left(e_{z}\right) \\
& O_{A_{1}^{-}, r=1}^{\left[J=0, m_{J}=0, P=-, \lambda_{V}=0, \lambda_{P}=0\right]}=O_{A_{1}^{-}, r=1}^{\left[J=0, m_{J}=0, L=1, S=1\right]}=O_{A_{1}^{-}, r=1} \\
& T_{1}^{+}: \\
& O_{T_{1}^{+}, r=1, n=1}=\mathrm{P}\left(e_{x}\right) V_{x}\left(-e_{x}\right)+\mathrm{P}\left(-e_{x}\right) V_{x}\left(e_{x}\right) \\
& O_{T_{1}^{+}, r=1, n=2}=\mathrm{P}\left(e_{y}\right) V_{x}\left(-e_{y}\right)+\mathrm{P}\left(-e_{y}\right) V_{x}\left(e_{y}\right)+\mathrm{P}\left(e_{z}\right) V_{x}\left(-e_{z}\right)+\mathrm{P}\left(-e_{z}\right) V_{x}\left(e_{z}\right) \\
& O_{T_{1}^{+}, r=1}^{\left[J=1, P=+, \lambda_{V}= \pm 1, \lambda_{P}=0\right]}=O_{T_{1}^{+}, r=1, n=2} \\
& O_{T_{1}^{+}, r=1}^{\left[J=1, P=+, \lambda_{V}=0, \lambda_{P}=0\right]}=O_{T_{1}^{+}, r=1, n=1}
\end{aligned}
$$




$$
\begin{aligned}
& O_{T_{1}^{+}, r=1}^{[J=1, L=0, S=1]}=O_{T_{1}^{+}, r=1, n=1}+O_{T_{1}^{+}, r=1, n=2} \\
& O_{T_{1}^{+}, r=1}^{[J=1, L=2, S=1]}=-2 O_{T_{1}^{+}, r=1, n=1}+O_{T_{1}^{+}, r=1, n=2} \\
& T_{1}^{-} \text {: } \\
& O_{T_{1}^{-}, r=1}=-\mathrm{P}\left(e_{y}\right) V_{z}\left(-e_{y}\right)+\mathrm{P}\left(-e_{y}\right) V_{z}\left(e_{y}\right)+\mathrm{P}\left(e_{z}\right) V_{y}\left(-e_{z}\right)-\mathrm{P}\left(-e_{z}\right) V_{y}\left(e_{z}\right) \\
& O_{T_{1}^{-}, r=1}^{\left[J=1, P=-, \lambda_{V}= \pm 1, \lambda_{P}=0\right]}=O_{T_{1}^{-}, r=1}^{[J=1, L=1, S=1]}=O_{T_{1}^{-}, r=1} \\
& T_{2}^{+} \text {: } \\
& O_{T_{2}^{+}, r=1}=\mathrm{P}\left(e_{y}\right) V_{x}\left(-e_{y}\right)+\mathrm{P}\left(-e_{y}\right) V_{x}\left(e_{y}\right)-\mathrm{P}\left(e_{z}\right) V_{x}\left(-e_{z}\right)-\mathrm{P}\left(-e_{z}\right) V_{x}\left(e_{z}\right) \\
& O_{T_{2}^{+}, r=1}^{\left[J=2, P=+, \lambda_{V}= \pm 1, \lambda_{P}=0\right]}=O_{T_{2}^{+}, r=1}^{[J=2, L=2, S=1]}=O_{T_{2}^{+}, r=1} \\
& T_{2}^{-} \text {: } \\
& O_{T_{2}^{-}, r=1}=\mathrm{P}\left(e_{y}\right) V_{z}\left(-e_{y}\right)-\mathrm{P}\left(-e_{y}\right) V_{z}\left(e_{y}\right)+\mathrm{P}\left(e_{z}\right) V_{y}\left(-e_{z}\right)-\mathrm{P}\left(-e_{z}\right) V_{y}\left(e_{z}\right) \\
& O_{T_{2}^{-}, r=1}^{\left[J=2, P=-, \lambda_{V}= \pm 1, \lambda_{P}=0\right]}=O_{T_{2}^{-}, r=1}^{[J=2, L=1, S=1]}=O_{T_{2}^{-}, r=1}^{[J=2, L=3, S=1]}=O_{T_{2}^{-}, r=1} \\
& E^{-} \text {: } \\
& O_{E^{-}, r=1}=\mathrm{P}\left(e_{x}\right) V_{x}\left(-e_{x}\right)-\mathrm{P}\left(-e_{x}\right) V_{x}\left(e_{x}\right)+\mathrm{P}\left(e_{y}\right) V_{y}\left(-e_{y}\right)-\mathrm{P}\left(-e_{y}\right) V_{y}\left(e_{y}\right) \\
& -2 \mathrm{P}\left(e_{z}\right) V_{z}\left(-e_{z}\right)+2 \mathrm{P}\left(-e_{z}\right) V_{z}\left(e_{z}\right) \\
& O_{E^{-}, r=1}^{\left[J=2, P=-, \lambda_{V}=0, \lambda_{P}=0\right]}=O_{E^{-}, r=1}^{[J=2, L=1, S=1]}=O_{E^{-}, r=1}^{[J=2, L=3, S=1]}=O_{E^{-}, r=1} \\
& O_{A_{1}^{+}}=O_{A_{2}^{+}}=O_{A_{2}^{-}}=O_{E^{+}}=0 .
\end{aligned}
$$

There is only one independent operator with $|p| \leq 1$ for each irrep (and given row), except for $T_{1}^{+}$. The result confirms, for example, that $O_{A_{1}^{-}, r=1}^{\left[J=0, P=-, \lambda_{V}=0\right]}=O_{A_{1}^{-}, r=1}$ (5.3) relates to a channel with negative parity and zero-helicity vector. Or that $O_{T_{2}^{-}, r=1}^{[J=2, L=1, S=1]}=$ $O_{T_{2}^{-}, r=1}$ (5.7) gives information on the channel with $J=2, L=1$ and $S=1$. Of course, certain states with higher $J$ and $L$ inevitably contribute to the same discrete irrep along the lines of table 1. For example states with $J=2, L=3, S=1$ also contribute to the same irrep $T_{2}^{-}$and the operators $O_{T_{2}^{-}, r=1}^{[J=2, L=1, S=1]}=O_{T_{2}^{-}, r=1}^{[J=2, L=3, S=1]}$ can not distinguish between $L=1$ and $L=3$.

The irrep $T_{1}^{+}$presents an interesting case where there are two independent interpolators $n=1,2$ at $|p|=1$. The result (5.4) indicates that $O_{T_{1}^{+}, n=1}$ is relevant for $\lambda_{V}=0$, while $O_{T_{1}^{+}, n=2}$ is relevant for $\left|\lambda_{V}\right|=1$. The expressions (5.4) also indicate which linear combinations of $O_{T_{1}^{+}, n}$ need to be employed to study $L=0$ or $L=2$ partial waves. Note that both partial waves contribute to the same $J^{P}=1^{+}$channel even in the continuum $P V$ scattering with $S=1$. Specific higher $J \geq 3$ and $L \geq 4$ lead to the same $T_{1}^{+}$operators (or their linear combinations). In the limit when partial waves $L \geq 4$ are negligible, we expect that $O_{T_{1}^{+}}^{[J=1, L=0, S=1]}$ and $O_{T_{1}^{+}}^{[J=1, L=2, S=1]}$ are valuable handles on lowest partial waves $L=0$ and 2 , respectively. This is expected to work better when the physical transition 
( $L=0, S=1) \leftrightarrow(L=2, S=1)$ in this channel is small, which is realized, for example, in the heavy-light meson observables due to the heavy quark symmetry. ${ }^{12}$

The correct number of independent interpolators for $|p|=1$ can be verified as follows. The total number can be obtained by counting different $P(p) V_{i}(-p)$, which span a basis with $6 \cdot 3=18$ terms ( 6 for directions of $p$ and 3 for polarization of $V$ ). This agrees with the number $5 \cdot 3+2+1=18$ of interpolators above in all rows ( 5 for number of independent $O_{T_{1,2}, r=1}$ and 3 for number of rows in $\left.T_{1,2}\right)$. The number of $P(1) V(-1)$ interpolators for each irrep also agrees with the fourth method [12], discussed in section 4.5. The table I in [12] indicates that $P(1)$ is in irrep $A_{2}$ of group $D i c_{4}$, while $V(-1)$ can be in $E_{2}$ or $A_{1}$ of $D i c_{4}$. The $P(1) V(-1)$ are combined to $A_{2} \otimes\left(E_{2} \oplus A_{1}\right)=\left(T_{1}^{+} \oplus T_{1}^{-} \oplus T_{2}^{+} \oplus T_{2}^{-}\right) \oplus\left(T_{1}^{+} \oplus A_{1}^{-} \oplus E^{-}\right)$ according to table III in [12]. This agrees with our interpolators above, so $T_{1}^{+}$indeed appears twice.

\section{$5.2 \quad P N, V N$ and $N N$ operators}

The explicit expressions for $|p|=0,1$ and all irreps are presented in appendix C. Several linearly independent operators $O_{\Gamma, r, n}$ typically arise for $V N$ and $N N$ in most of irreps. The number of linearly independent operators in a given irrep is the same as the number of different $(L, S)$ combinations that can contribute to given $J^{P}$ in the continuum limit (this applies for $J \leq 3 / 2$ which are contained in a single irrep according to table 1 ). The partial-wave and helicity operators indicate which linear combinations of $O_{\Gamma, n}$ are most relevant to study scattering with given $(J, L, S)$ or given $\left(J, P, \lambda_{1}, \lambda_{2}\right)$.

\section{Conclusions}

We consider three different methods to construct two-hadron interpolators where one or both hadrons carry spin. The focus is on the case with total-momentum zero where parity $P$ is a good quantum number. The correct transformation properties are proven for all three methods. The projection method is a general mathematical tool which leads to one or several operators $O_{\Gamma, r, n}$ that transform according to given irrep $\Gamma$ and row $r$, but it does not give much insight on the underlying continuum quantum numbers. The partial-wave and the helicity methods indicate which linear combinations $O_{\Gamma, r, n}$ of various $n$ have to be employed in order to hopefully enhance couplings to the states with desired continuum quantum numbers. The partial-wave method renders operators $O_{\Gamma, r}^{[J, L, S]}$ with enhanced couplings to two-hadron states in partial wave $L$, total spin $S$ and total angular momentum $J$. The helicity method provides operators $O_{\Gamma, r}^{\left[J, P, \lambda_{1}, \lambda_{2}\right]}$ where each hadron has good helicity $\lambda_{1,2}$. The quality of these enhancements depends on how close one is to the physical limit, the smallness of the disregarded higher partial waves, and on the magnitude of the physical transitions between various $(L, S)$ for a given $J^{P}$.

Explicit expressions for $P V, P N, V N$ and $N N$ operators with lowest two relative momenta are provided in all irreducible representations. We verified that all three methods

\footnotetext{
${ }^{12}$ One $Q \bar{q}$ state with $J^{P}=1^{+}$decays only via $L=0$ in the $m_{Q} \rightarrow \infty$ limit $[21]$, and $O_{T_{1}^{+}}^{[J=1, L=0, S=1]}$ was used to consider $D_{1}(2430) \rightarrow D^{*} \pi$ in [22]. The other $J^{P}=1^{+}$state decays only via $L=2$ and is narrow.
} 
lead to consistent results, where partial-wave and helicity methods are particularly helpful for physics interpretation. The operators shall be valuable to simulate the scattering of particles with spin in desired channels using quantum field theory on the lattice.

\section{Acknowledgments}

We want to thank kindly R. Briceño, J. Dudek, R. Edwards, A. Nicholson, M. Padmanath and A. Walker-Loud for valuable discussions. We are especially grateful to M. Padmanath for insightful discussions on the construction of $P N$ interpolators. This work is supported in part by the Slovenian Research Agency ARRS, and by the Austrian science Found project FWF:I1313-N27. S.P. acknowledges support from U.S. Department of Energy Contract No. DE-AC05-06OR23177, under which Jefferson Science Associates, LLC, manages and operates Jefferson Laboratory.

\section{A Proofs of transformation properties for operators}

\section{A.1 Projection method}

The arbitrary operator $H^{(1), a} H^{(2), a}$ in (4.1) is a linear combination of $O_{\Gamma^{\prime}, r^{\prime}}$ and the projector projects out $O_{\Gamma, r}$ :

$$
\begin{aligned}
\sum_{\tilde{R} \in G} T_{r, r}^{\Gamma}(\tilde{R}) \tilde{R} O_{\Gamma^{\prime}, r^{\prime}} \tilde{R}^{-1} & =\sum_{\tilde{R}} T_{r, r}^{\Gamma}(\tilde{R}) \sum_{r^{\prime \prime}} T_{r^{\prime \prime}, r^{\prime}}^{\Gamma^{\prime}}(\tilde{R})^{*} O_{\Gamma^{\prime}, r^{\prime \prime}} \\
& =\sum_{r^{\prime \prime}}\left[\sum_{\tilde{R}} T_{r, r}^{\Gamma}(\tilde{R}) T_{r^{\prime \prime}, r^{\prime}}^{\Gamma^{\prime}}(\tilde{R})^{*}\right] O_{\Gamma^{\prime}, r^{\prime \prime}} \\
& =\frac{\operatorname{dim}_{\Gamma}}{n_{G}} \sum_{r^{\prime \prime}} \delta_{\Gamma \Gamma^{\prime}} \delta_{r r^{\prime \prime}} \delta_{r r^{\prime}} O_{\Gamma^{\prime}, r^{\prime \prime}}=\delta_{\Gamma \Gamma^{\prime}} \delta_{r r^{\prime}} O_{\Gamma, r}
\end{aligned}
$$

where (3.2) and the orthogonality theorem [19] were used, while $n_{G}$ is number of group elements.

\section{A.2 Helicity method}

Let us verify that annihilation operator (4.6) transforms under rotation $R_{a} \in O^{(2)}$ as (3.1), where indices $|p|$ and $\lambda$ are omitted for brevity

$$
\begin{aligned}
R_{a} O^{J, m_{J}, \lambda_{1}, \lambda_{2}} R_{a}^{-1} & =\sum_{R \in O^{(2)}} D_{m_{J}, \lambda}^{J}(R) R_{a} R H_{\lambda_{1}}^{h}(p) H_{\lambda_{2}}^{h}(-p) R^{-1} R_{a}^{-1} \\
& =\sum_{R \in O^{(2)}} D_{m_{J}, \lambda}^{J}\left(R_{a}^{-1} R^{\prime}\right) R^{\prime} H_{\lambda_{1}}^{h}(p) H_{\lambda_{2}}^{h}(-p) R^{\prime-1} \\
& =\sum_{R^{\prime} \in O^{(2)}} \sum_{m_{J}^{\prime}} D_{m_{J}, m_{J}^{\prime}}^{J}\left(R_{a}^{-1}\right) D_{m_{J}^{\prime}, \lambda}^{J}\left(R^{\prime}\right) R^{\prime} H_{\lambda_{1}}^{h}(p) H_{\lambda_{2}}^{h}(-p) R^{\prime-1} \\
& =\sum_{m_{J}^{\prime}} D_{m_{J}, m_{J}^{\prime}}^{J}\left(R_{a}^{-1}\right) \sum_{R^{\prime} \in O^{(2)}} D_{m_{J}^{\prime}, \lambda}^{J}\left(R^{\prime}\right) R^{\prime} H_{\lambda_{1}}^{h}(p) H_{\lambda_{2}}^{h}(-p) R^{\prime-1} \\
& =\sum_{m_{J}^{\prime}} D_{m_{J}, m_{J}^{\prime}}^{J}\left(R_{a}^{-1}\right) O^{J, m_{J}^{\prime}, \lambda_{1}, \lambda_{2}},
\end{aligned}
$$


where we defined $R^{\prime}=R_{a} R$ and used (2.3).

\section{A.3 Partial-wave method}

Here we verify that operator (4.11) transforms under rotation $R_{a} \in O^{(2)}$ as (3.1). First the summation $R \in O$ (4.11) can be replaced by $R \in O^{(2)}$ since both have the same effect on $p^{13}$

$$
\begin{aligned}
R_{a} O^{J, m_{J}, L, S} R_{a}^{-1}= & \sum_{m_{L}, m_{S}, m_{s 1}, m_{s 2}} C_{L m_{L}, S m_{S}}^{J m_{J}} C_{s_{1} m_{s 1}, s_{2} m_{s 2}}^{S m_{S}} \sum_{R \in O^{(2)}} Y_{L m_{L}}^{*}(\hat{R p}) R_{a} H_{m_{s 1}}(R p) H_{m_{s 2}}(-R p) R_{a}^{-1} \\
= & \sum_{m_{L}, m_{S}, m_{s 1}, m_{s 2}} C_{L m_{L}, S m_{S}}^{J m_{J}} C_{s_{1} m_{s 1}, s_{2} m_{s 2}}^{S m_{S}} \sum_{R \in O_{h}} Y_{L m_{L}}^{*}(\hat{R p}) \\
& \times \sum_{m_{s 1}^{\prime}} D_{m_{s 1} m_{s 1}^{\prime}}^{s_{1}}\left(R_{a}^{-1}\right) H_{m_{s 1}^{\prime}}\left(R_{a} R p\right) \sum_{m_{s 2}^{\prime}} D_{m_{s 2} m_{s 2}^{\prime}}^{s_{2}}\left(R_{a}^{-1}\right) H_{m_{s 2}^{\prime}}\left(-R_{a} R p\right), \quad \text { (A.3) }
\end{aligned}
$$

where (2.2) was used. We intruduce introduce $R^{\prime} \equiv R_{a} R$, which is also an element of $O^{(2)}$, and use

$$
Y_{L m_{L}}^{*}(R p)=Y_{L m_{L}}^{*}\left(R_{a}^{-1}\left(R^{\prime} p\right)\right)=\sum_{m_{L}^{\prime}} D_{m_{L} m_{L}^{\prime}}^{L}\left(R_{a}^{-1}\right) Y_{L m_{L}^{\prime}}^{*}\left(R^{\prime} p\right)
$$

where the second step follows from $Y_{L m_{L}}^{*}\left(R_{1} p\right)=\sum_{m_{L}^{\prime}} D_{m_{L} m_{L}^{\prime}}^{L}\left(R_{1}\right) Y_{L m_{L}^{\prime}}^{*}(p) .{ }^{14}$

Pairs of D's are combined

$$
\begin{aligned}
D_{m_{s 1} m_{s 1}^{\prime}}^{s_{1}}\left(R_{a}^{-1}\right) D_{m_{s 2} m_{s 2}^{\prime}}^{s_{2}}\left(R_{a}^{-1}\right) & =\sum_{\tilde{S}, \tilde{m}_{S}, m_{S}^{\prime}} C_{s_{1} m_{s 1}, s_{2} m_{s 2}}^{\tilde{\tilde{S}} \tilde{\tilde{m}}_{S}} C_{s_{1} m_{s 1}^{\prime}, s_{2} m_{s 2}^{\prime}}^{\tilde{S}, m_{S}^{\prime}} D_{\tilde{m}_{S} m_{S}^{\prime}}^{\tilde{S}}\left(R_{a}^{-1}\right) \\
D_{m_{L} m_{L}^{\prime}}^{L}\left(R_{a}^{-1}\right) D_{\tilde{m}_{S} m_{S}^{\prime}}^{\tilde{S}}\left(R_{a}^{-1}\right) & =\sum_{\tilde{J}, \tilde{m}_{J}, m_{J}^{\prime}} C_{L m_{L}, \tilde{S} \tilde{m}_{S}}^{\tilde{J}_{S} \tilde{m}_{J}} C_{L m_{L}^{\prime}, \tilde{S} m_{S}^{\prime}}^{\tilde{J}, m_{J}^{\prime}} D_{\tilde{m}_{J} m_{J}^{\prime}}^{\tilde{J}}\left(R_{a}^{-1}\right)
\end{aligned}
$$

and pairs of Clebsch-Gordan coefficients with the same subscripts are summed to get

$$
\sum_{m_{s 1}, m_{s 2}} C_{s_{1} m_{s 1}, m_{2} m_{s 2}}^{S m_{S}} C_{s_{1} m_{s 1}, s_{2} m_{s 2}}^{\tilde{S}, \tilde{m}_{S}}=\delta_{m_{S}, \tilde{m}_{S}} \delta_{S, \tilde{S}}, \quad \sum_{m_{L}, m_{S}} C_{L m_{L}, S m_{S}}^{J m_{J}} C_{L m_{L}, S m_{S}}^{\tilde{J}, \tilde{m}_{J}}=\delta_{m_{J}, \tilde{m}_{J}} \delta_{J, \tilde{J}}
$$

Inserting all this to (A.3) one has

$$
\begin{aligned}
& R_{a} O^{J, m_{J}, L, S} R_{a}^{-1}= \\
& =\sum_{m_{J}^{\prime}} D_{m_{J} m_{J}^{\prime}}^{J}\left(R_{a}^{-1}\right) \sum_{m_{L}^{\prime}, m_{S}^{\prime}, m_{s 1}^{\prime}, m_{s 2}^{\prime}} C_{L m_{L}^{\prime}, S m_{S}^{\prime}}^{J m_{J}^{\prime}} C_{s_{1} m_{s 1}^{\prime}, s_{2} m_{s 2}^{\prime}}^{S m_{2}^{\prime}} \sum_{R^{\prime} \in O^{(2)}} Y_{L m_{L}^{\prime}}^{*}\left(\hat{R^{\prime} p} p H_{m_{s 1}^{\prime}}\left(R^{\prime} p\right) H_{m_{s 2}^{\prime}}\left(-R^{\prime} p\right)\right. \\
& =\sum_{m_{J}^{\prime}} D_{m_{J} m_{J}^{\prime}}^{J}\left(R_{a}^{-1}\right) O^{J, m_{J}^{\prime}, L, S}
\end{aligned}
$$

as needed to verify (3.1). In the last step $R \in O^{(2)}$ was replaced by back to $R \in O$ in (4.11) as both have the same effect on $p$.

\footnotetext{
${ }^{13}$ An irrelevant overall factor $1 / 2$ that might arise is here omitted.

${ }^{14}$ See for example appendix C of [Messiah]. This relation depends on the convention of Wigner D matrix and we have explicitly verified it for employed convention given in the appendix B.1.
} 


\section{B Technicalities}

\section{B.1 Wigner D-matrices}

We employ the conventional definition of Wigner D-matrix, which renders (2.1) and is used for example in $[13,14]$. That convention differs from the definition in Wigner's book [23], which is incorporated in Wolfram's Mathematica [24]. ${ }^{15}$ The employed $D^{j}$ for the rotation $R$ that is consistent with (2.1) is

$$
\begin{aligned}
& D_{m, m^{\prime}}^{j}\left[R_{\alpha \beta \gamma}^{\omega}\right]=F \cdot \text { WignerD }\left[\left\{j, m, m^{\prime}\right\},-\alpha,-\beta,-\gamma\right], \\
& F=\left\{\begin{array}{l}
1: j=\text { integer } \\
\pm 1: j=\text { halfinteger; choice of sign in paragraph below, } \mathrm{F}(\omega+2 \pi)=-\mathrm{F}(\omega) .
\end{array}\right.
\end{aligned}
$$

WignerD denotes the Mathematica function and $-\pi \leq \omega<3 \pi$ is the angle of rotation $R$ around $\vec{n}$ in positive direction. The rotations for $\pi \leq \omega<3 \pi$ are present only in the double cover group.

The Euler angles of $R$ can be obtained with Mathematica as $\{\alpha, \beta, \gamma\}=$ EulerAngles $[T]$ with $T=\exp (-i \vec{n} \vec{J} \omega)$ and $\left(J_{k}\right)_{i j}=-i \epsilon_{i j k}$ (2.5). They are the same for $\omega$ and $\omega+2 \pi$, and the only difference between these two rotations is given by the factor $F$. The sign of $F= \pm 1$ for half-integer $j$ and $\pi \leq \omega<\pi$ can be arbitrarily chosen for rotations $i=1, \ldots, 24$ in table A.1 of [16]: we choose it so that $D^{1 / 2}=\exp \left(-\frac{i}{2} \vec{n} \vec{\sigma} \omega\right)$, which gives $F=-1$ for $i=2,4,12,16,18,20,21,23,24$ and $F=1$ for remaining fifteen. The sign of next 24 elements of $O^{2}$ with $\pi \leq \omega<3 \pi$ is fixed by $F(\omega+2 \pi)=-F(\omega)$. The resulting operators do not depend on the choice of the sign in $F$, except for irrelevant multiplicative factor. ${ }^{16}$

\section{B.2 Transformations of basis vectors and components}

Let the hadron operator $H$ be a linear combination of operators $H_{m}$ ( $m$ can stand for $m_{s}$ or $i=x, y, z)$

$$
H=\sum c_{m} H_{m}=\left(\begin{array}{l}
c_{1} \\
c_{2} \\
\ldots
\end{array}\right),
$$

where $H_{m}$ are the basis vectors and $c_{m}$ the coefficients. If the basis vectors $H_{m}$ transform according to

$$
R H_{m} R^{-1}=\sum_{\tilde{m}} M_{\tilde{m} m} H_{\tilde{m}}
$$

(which can be deduced from (2.1), (2.5)) then

$$
R H R^{-1}=\sum_{m} c_{m} R H_{m} R^{-1}=\sum_{m, \tilde{m}} c_{m} M_{\tilde{m} m} H_{\tilde{m}}=\sum_{\tilde{m}} c_{\tilde{m}}^{\prime} H_{\tilde{m}}
$$

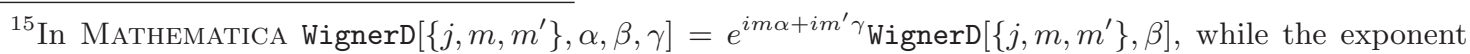
has different sign for a more conventional definition employed here.

${ }^{16}$ Consider for example $N V$ helicity operators (4.8): each term of sum in $R$ contains $F^{2}=1$, where one $F= \pm 1$ is present in $D^{J}$ and the same $F$ is present in the rotation of $N$ (both related to half-integer spin).
} 
and the coefficients $c$ transform like

$$
c_{\tilde{m}}^{\prime}=\sum_{m} M_{\tilde{m} m} c_{m}
$$

\section{B.3 Two bases for vectors}

The vector states $V^{\dagger}|0\rangle=|V\rangle=A_{x}\left|V_{x}\right\rangle+A_{y}\left|V_{y}\right\rangle+A_{z}\left|V_{z}\right\rangle$ with coefficients $A^{17}$

$$
\left(\begin{array}{c}
A_{x} \\
A_{y} \\
A_{z}
\end{array}\right)_{m_{J}=1}=\frac{1}{\sqrt{2}}\left(\begin{array}{c}
-1 \\
-i \\
0
\end{array}\right), \quad\left(\begin{array}{c}
A_{x} \\
A_{y} \\
A_{z}
\end{array}\right)_{m_{J}=0}=\left(\begin{array}{l}
0 \\
0 \\
1
\end{array}\right), \quad\left(\begin{array}{c}
A_{x} \\
A_{y} \\
A_{z}
\end{array}\right)_{m_{J}=-1}=\frac{1}{\sqrt{2}}\left(\begin{array}{c}
1 \\
-i \\
0
\end{array}\right)
$$

have good $S_{z}$ at rest, where $\left(S_{k}\right)_{i j}=-i \epsilon_{i j k}$

$$
S_{z}\left(\begin{array}{l}
A_{x} \\
A_{y} \\
A_{z}
\end{array}\right)_{m_{J}}=-i\left(\begin{array}{ccc}
0 & 1 & 0 \\
-1 & 0 & 0 \\
0 & 0 & 0
\end{array}\right)\left(\begin{array}{l}
A_{x} \\
A_{y} \\
A_{z}
\end{array}\right)_{m_{J}}=m_{J}\left(\begin{array}{c}
A_{x} \\
A_{y} \\
A_{z}
\end{array}\right) .
$$

The annihilation operators $V(2.4)$ are obtained by hermitian conjugation of $V^{\dagger}$, so annihilation operators have complex-conjugated coefficients with respect to the states above.

\section{B.4 Subduction matrices $\mathcal{S}$}

The subduction coefficients in (4.10) depend on the conventions for the rows of the irrep. Our conventions agree with appendix A of [16] for all irreps. This also agrees with rows [8, $20]$ for all irreps except for $T_{1,2}$. Our rows are proportional to the entries $(x, y, z)$ and $(y z, z x, x y)$ for $T_{1}$ and $T_{2}$, respectively, while they are related to $\left(Y_{1,1}, Y_{1,0}, Y_{1,-1}\right)$ and $\left(Y_{2,1}, \frac{1}{2}\left(Y_{2,2}-Y_{2,-2}\right), Y_{2,1}\right)$ in $[8,20,25]$. We employ all subduction matrices $\mathcal{S}$ from [8, 20] except for $T_{1,2}$ which are deduced from [20]:

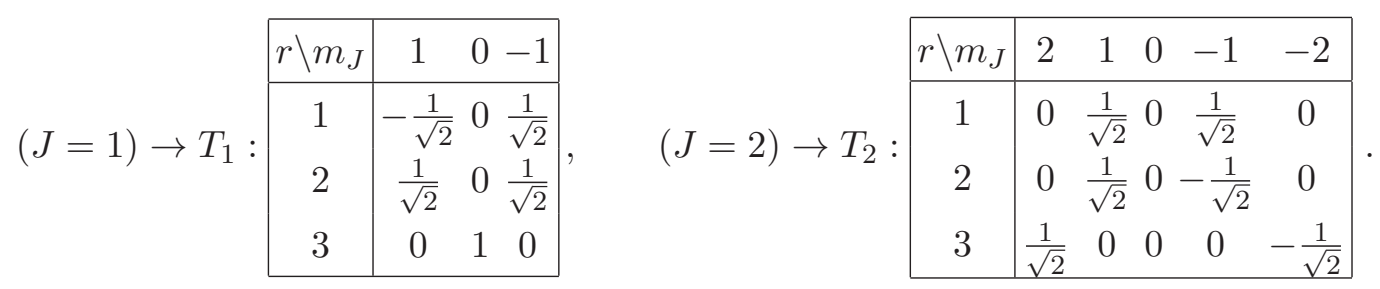

\section{Expressions for two-hadron operators}

\section{C.1 PN operators}

C.1.1 $|p|=0$

$$
\begin{aligned}
& G_{1}^{-}: \\
& O_{G_{1}^{-}, r=1}=N_{\frac{1}{2}}(0) \mathrm{P}(0) \\
& O_{G_{1}^{-}, r=1}^{\left[J=\frac{1}{2}, L=0, S=\frac{1}{2}\right]}=O_{G_{1}^{-}, r=1}
\end{aligned}
$$

Operators for other irreps are equal to 0 .

\footnotetext{
${ }^{17}$ Coefficients are in agreement with $Y_{11} \propto-x-i y, Y_{10} \propto z, Y_{1-1} \propto x-i y$.
} 
C.1.2 $|p|=1$

$G_{1}^{+}$:

$\begin{aligned} O_{G_{1}^{+}, r=1}= & N_{-\frac{1}{2}}\left(-e_{x}\right) \mathrm{P}\left(e_{x}\right)-N_{-\frac{1}{2}}\left(e_{x}\right) \mathrm{P}\left(-e_{x}\right)-i N_{-\frac{1}{2}}\left(-e_{y}\right) \mathrm{P}\left(e_{y}\right)+i N_{-\frac{1}{2}}\left(e_{y}\right) \mathrm{P}\left(-e_{y}\right) \\ & +N_{\frac{1}{2}}\left(-e_{z}\right) \mathrm{P}\left(e_{z}\right)-N_{\frac{1}{2}}\left(e_{z}\right) \mathrm{P}\left(-e_{z}\right)\end{aligned}$

$O_{G_{1}^{+}, r=1}^{\left[J=\frac{1}{2}, m_{J}=\frac{1}{2}, P=+, \lambda_{N}= \pm \frac{1}{2}, \lambda_{P}=0\right]}=O_{G_{1}^{-}, r=1}^{\left[J=\frac{1}{2}, m_{J}=\frac{1}{2}, L=1, S=\frac{1}{2}\right]}=O_{G_{1}^{+}, r=1}$

$O_{\mathrm{G}_{2}^{+}}=O_{\mathrm{G}_{2}^{-}}=0$

$G_{1}^{-}$:

$O_{G_{1}^{-}, r=1}=N_{\frac{1}{2}}\left(-e_{x}\right) \mathrm{P}\left(e_{x}\right)+N_{\frac{1}{2}}\left(e_{x}\right) \mathrm{P}\left(-e_{x}\right)+N_{\frac{1}{2}}\left(-e_{y}\right) \mathrm{P}\left(e_{y}\right)+N_{\frac{1}{2}}\left(e_{y}\right) \mathrm{P}\left(-e_{y}\right)$ $+N_{\frac{1}{2}}\left(-e_{z}\right) \mathrm{P}\left(e_{z}\right)+N_{\frac{1}{2}}\left(e_{z}\right) \mathrm{P}\left(-e_{z}\right)$

$O_{G_{1}^{-}, r=1}^{\left[J=\frac{1}{2}, m_{J}=\frac{1}{2}, P=-, \lambda_{N}= \pm \frac{1}{2}, \lambda_{P}=0\right]}=O_{G_{1}^{-}, r=1}^{\left[J=\frac{1}{2}, m_{J}=\frac{1}{2}, L=0, S=\frac{1}{2}\right]}=O_{G_{1}^{-}, r=1}$

$\mathrm{H}^{+}$

$O_{H^{+}, r=1}=-i N_{\frac{1}{2}}\left(-e_{x}\right) \mathrm{P}\left(e_{x}\right)+i N_{\frac{1}{2}}\left(e_{x}\right) \mathrm{P}\left(-e_{x}\right)-N_{\frac{1}{2}}\left(-e_{y}\right) \mathrm{P}\left(e_{y}\right)+N_{\frac{1}{2}}\left(e_{y}\right) \mathrm{P}\left(-e_{y}\right)$

$O_{H^{+}, r=1}^{\left[J=\frac{3}{2}, m_{J}=\frac{3}{2}, P=+, \lambda_{N}= \pm \frac{1}{2}, \lambda_{P}=0\right]}=O_{H^{+}, r=1}^{\left[J=\frac{5}{2}, P=+, \lambda_{N}= \pm \frac{1}{2}, \lambda_{P}=0\right]}=O_{H^{+}, r=1}$

$O_{H^{+}, r=1}^{\left[J=\frac{3}{2}, m_{J}=\frac{3}{2}, L=1, S=\frac{1}{2}\right]}=O_{H^{+}, r=1}^{\left[J=\frac{5}{2}, L=3, S=\frac{1}{2}\right]}=O_{H^{+}, r=1}$

$\mathrm{H}^{-}$:

$O_{H^{-}, r=1}=N_{-\frac{1}{2}}\left(-e_{x}\right) \mathrm{P}\left(e_{x}\right)+N_{-\frac{1}{2}}\left(e_{x}\right) \mathrm{P}\left(-e_{x}\right)-N_{-\frac{1}{2}}\left(-e_{y}\right) \mathrm{P}\left(e_{y}\right)-N_{-\frac{1}{2}}\left(e_{y}\right) \mathrm{P}\left(-e_{y}\right)$

$O_{H^{-}, r=1}^{\left[J=\frac{3}{2}, m_{J}=\frac{3}{2}, P=-, \lambda_{N}= \pm \frac{1}{2}, \lambda_{P}=0\right]}=O_{H^{-}, r=1}^{\left[J=\frac{5}{2}, P=-, \lambda_{N}= \pm \frac{1}{2}, \lambda_{P}=0\right]}=O_{H^{-}, r=1}$

$O_{H^{-}, r=1}^{\left[J=\frac{3}{2}, m_{J}=\frac{3}{2}, L=2, S=\frac{1}{2}\right]}=O_{H^{-}, r=1}^{\left[J=\frac{5}{2}, L=2, S=\frac{1}{2}\right]}=O_{H^{-}, r=1}$

The $O_{G_{1}^{+}}$for $|p|=1$ was more recently used to simulate the $N \pi$ scattering in the $J^{P}=1 / 2^{+}$ channel [26], where the Roper resonance was found in experiment.

\section{C.2 NV operators}

\section{C.2.1 $|p|=0$}

$$
\begin{aligned}
& G_{1}^{-}: \\
& O_{G_{1}^{-}, r=1}=N_{\frac{1}{2}}(0) V_{z}(0)+N_{-\frac{1}{2}}(0)\left(V_{x}(0)-i V_{y}(0)\right) \\
& O_{G_{1}^{-}, r=1}^{\left[J=\frac{1}{2}, m_{J}=\frac{1}{2}, L=0, S=\frac{1}{2}\right]}=O_{G_{1}^{-}, r=1}
\end{aligned}
$$




$$
\begin{aligned}
& H^{-}: \\
& O_{H^{-}, r=1}=N_{\frac{1}{2}}(0)\left(V_{x}(0)-i V_{y}(0)\right) \\
& O_{H^{-}, r=1}^{\left[J=\frac{3}{2}, L=0, S=\frac{3}{2}\right]}=O_{H^{-}, r=1}
\end{aligned}
$$

Operators for other irreps are equal to 0.

\section{C.2.2 $|p|=1$}

$\mathrm{G}_{1}^{+}$:

$$
\begin{aligned}
& O_{G_{1}^{+}, r=1, n=1}= N_{\frac{1}{2}}\left(e_{x}\right) V_{y}\left(-e_{x}\right)-N_{\frac{1}{2}}\left(-e_{x}\right) V_{y}\left(e_{x}\right)+i N_{-\frac{1}{2}}\left(e_{x}\right) V_{z}\left(-e_{x}\right)-i N_{-\frac{1}{2}}\left(-e_{x}\right) V_{z}\left(e_{x}\right) \\
&-N_{\frac{1}{2}}\left(e_{y}\right) V_{x}\left(-e_{y}\right)+N_{\frac{1}{2}}\left(-e_{y}\right) V_{x}\left(e_{y}\right)+N_{-\frac{1}{2}}\left(e_{y}\right) V_{z}\left(-e_{y}\right)-N_{-\frac{1}{2}}\left(-e_{y}\right) V_{z}\left(e_{y}\right) \\
&-i N_{-\frac{1}{2}}\left(e_{z}\right) V_{x}\left(-e_{z}\right)+i N_{-\frac{1}{2}}\left(-e_{z}\right) V_{x}\left(e_{z}\right)-N_{-\frac{1}{2}}\left(e_{z}\right) V_{y}\left(-e_{z}\right)+N_{-\frac{1}{2}}\left(-e_{z}\right) V_{y}\left(e_{z}\right) \\
& O_{G_{1}^{+}, r=1, n=2}= N_{\frac{1}{2}}\left(e_{x}\right) V_{x}\left(-e_{x}\right)-N_{\frac{1}{2}}\left(-e_{x}\right) V_{x}\left(e_{x}\right)+N_{\frac{1}{2}}\left(e_{y}\right) V_{y}\left(-e_{y}\right)-N_{\frac{1}{2}}\left(-e_{y}\right) V_{y}\left(e_{y}\right) \\
&+N_{\frac{1}{2}}\left(e_{z}\right) V_{z}\left(-e_{z}\right)-N_{\frac{1}{2}}\left(-e_{z}\right) V_{z}\left(e_{z}\right) \\
& O_{G_{1}^{+}, r=1}^{\left[J=\frac{1}{2}, P=+, \lambda_{N}=\frac{1}{2}, \lambda_{V}=1\right]}=O_{G_{1}^{+}, r=1}^{\left[J=\frac{1}{2}, P=+, \lambda_{N}=-\frac{1}{2}, \lambda_{V}=-1\right]}=O_{G_{1}^{+}, r=1, n=1} \\
& O_{G_{1}^{+}, r=1}^{\left[J=\frac{1}{2}, P=-, \lambda_{N}= \pm \frac{1}{2}, \lambda_{V}=0\right]}=O_{G_{1}^{+}, r=1, n=2} \\
& O_{G_{1}^{+}, r=1}^{\left[J=\frac{1}{2}, L=1, S=\frac{1}{2}\right]}=-i O_{G_{1}^{+}, r=1, n=1}-O_{G_{1}^{+}, r=1, n=2} \\
& O_{G_{1}^{+}, r=1}^{\left[J=\frac{1}{2}, L=1, S=\frac{3}{2}\right]}=-i O_{G_{1}^{+}, r=1, n=1}+2 O_{G_{1}^{+}, r=1, n=2}
\end{aligned}
$$

$\mathrm{G}_{1}^{-}$:

$$
\begin{aligned}
O_{G_{1}^{-}, r=1, n=1}= & N_{-\frac{1}{2}}\left(e_{x}\right) V_{y}\left(-e_{x}\right)+N_{-\frac{1}{2}}\left(-e_{x}\right) V_{y}\left(e_{x}\right)+i N_{\frac{1}{2}}\left(e_{x}\right) V_{z}\left(-e_{x}\right)+i N_{\frac{1}{2}}\left(-e_{x}\right) V_{z}\left(e_{x}\right) \\
& +i N_{-\frac{1}{2}}\left(e_{y}\right) V_{x}\left(-e_{y}\right)+i N_{-\frac{1}{2}}\left(-e_{y}\right) V_{x}\left(e_{y}\right)+i N_{\frac{1}{2}}\left(e_{y}\right) V_{z}\left(-e_{y}\right)+i N_{\frac{1}{2}}\left(-e_{y}\right) V_{z}\left(e_{y}\right) \\
& +i N_{-\frac{1}{2}}\left(e_{z}\right) V_{x}\left(-e_{z}\right)+i N_{-\frac{1}{2}}\left(-e_{z}\right) V_{x}\left(e_{z}\right)+N_{-\frac{1}{2}}\left(e_{z}\right) V_{y}\left(-e_{z}\right)+N_{-\frac{1}{2}}\left(-e_{z}\right) V_{y}\left(e_{z}\right) \\
O_{G_{1}^{-}, r=1, n=2}= & N_{-\frac{1}{2}}\left(e_{x}\right) V_{x}\left(-e_{x}\right)+N_{-\frac{1}{2}}\left(-e_{x}\right) V_{x}\left(e_{x}\right)-i N_{-\frac{1}{2}}\left(e_{y}\right) V_{y}\left(-e_{y}\right)-i N_{-\frac{1}{2}}\left(-e_{y}\right) V_{y}\left(e_{y}\right) \\
& +N_{\frac{1}{2}}\left(e_{z}\right) V_{z}\left(-e_{z}\right)+N_{\frac{1}{2}}\left(-e_{z}\right) V_{z}\left(e_{z}\right)
\end{aligned}
$$$$
O_{G_{1}^{-}, r=1}^{\left[J=\frac{1}{2}, P=-, \lambda_{N}=-\frac{1}{2}, \lambda_{V}=-1\right]}=O_{G_{1}^{-}, r=1}^{\left[J=\frac{1}{2}, P=-, \lambda_{N}=\frac{1}{2}, \lambda_{V}=1\right]}=O_{G_{1}^{-}, r=1, n=1}
$$$$
O_{G_{1}^{-}, r=1}^{\left[J, \frac{1}{2}, P=-, \lambda_{N}= \pm \frac{1}{2}, \lambda_{V}=0\right]}=O_{G_{1}^{-}, r=1, n=2}
$$$$
O_{G_{1}^{-}, r=1}^{\left[J=\frac{1}{2}, L=0, S=\frac{1}{2}\right]}=-i O_{G_{1}^{-}, r=1, n=1}+O_{G_{1}^{-}, r=1, n=2}
$$$$
O_{G_{1}^{-}, r=1}^{\left[J=\frac{1}{2}, L=2, S=\frac{3}{2}\right]}=-i O_{G_{1}^{-}, r=1, n=1}-2 O_{G_{1}^{-}, r=1, n=2}
$$

$\mathrm{G}_{2}^{+}$:

$$
\begin{aligned}
& O_{G_{2}^{+}, r=1}= N_{\frac{1}{2}}\left(e_{x}\right) V_{y}\left(-e_{x}\right)-N_{\frac{1}{2}}\left(-e_{x}\right) V_{y}\left(e_{x}\right)-i N_{-\frac{1}{2}}\left(e_{x}\right) V_{z}\left(-e_{x}\right)+i N_{-\frac{1}{2}}\left(-e_{x}\right) V_{z}\left(e_{x}\right) \\
&+N_{\frac{1}{2}}\left(e_{y}\right) V_{x}\left(-e_{y}\right)-N_{\frac{1}{2}}\left(-e_{y}\right) V_{x}\left(e_{y}\right)+N_{-\frac{1}{2}}\left(e_{y}\right) V_{z}\left(-e_{y}\right)-N_{-\frac{1}{2}}\left(-e_{y}\right) V_{z}\left(e_{y}\right) \\
&-i N_{-\frac{1}{2}}\left(e_{z}\right) V_{x}\left(-e_{z}\right)+i N_{-\frac{1}{2}}\left(-e_{z}\right) V_{x}\left(e_{z}\right)+N_{-\frac{1}{2}}\left(e_{z}\right) V_{y}\left(-e_{z}\right)-N_{-\frac{1}{2}}\left(-e_{z}\right) V_{y}\left(e_{z}\right) \\
& O_{G_{2}^{2}, r=1}^{\left[J=\frac{5}{2}, P=+, \lambda_{N}=-\frac{1}{2}, \lambda_{V}=1\right]}=O_{G_{2}^{+}, r=1}^{\left[J=\frac{5}{2}, P=+, \lambda_{N}=\frac{1}{2}, \lambda_{V}=-1\right]}=O_{G_{2}^{[}, r=1}^{\left[J=\frac{5}{2}, L=1, S=\frac{3}{2}\right]}=O_{G_{2}^{+}, r=1}
\end{aligned}
$$


$\mathrm{G}_{2}^{-}$:

$O_{G_{2}^{-}, r=1}=N_{-\frac{1}{2}}\left(e_{x}\right) V_{y}\left(-e_{x}\right)+N_{-\frac{1}{2}}\left(-e_{x}\right) V_{y}\left(e_{x}\right)-i N_{\frac{1}{2}}\left(e_{x}\right) V_{z}\left(-e_{x}\right)-i N_{\frac{1}{2}}\left(-e_{x}\right) V_{z}\left(e_{x}\right)$ $-i N_{-\frac{1}{2}}\left(e_{y}\right) V_{x}\left(-e_{y}\right)-i N_{-\frac{1}{2}}\left(-e_{y}\right) V_{x}\left(e_{y}\right)+i N_{\frac{1}{2}}\left(e_{y}\right) V_{z}\left(-e_{y}\right)+i N_{\frac{1}{2}}\left(-e_{y}\right) V_{z}\left(e_{y}\right)$ $+i N_{-\frac{1}{2}}\left(e_{z}\right) V_{x}\left(-e_{z}\right)+i N_{-\frac{1}{2}}\left(-e_{z}\right) V_{x}\left(e_{z}\right)-N_{-\frac{1}{2}}\left(e_{z}\right) V_{y}\left(-e_{z}\right)-N_{-\frac{1}{2}}\left(-e_{z}\right) V_{y}\left(e_{z}\right)$

$O_{G_{2}^{-}, r=1}^{\left[J=\frac{5}{2}, P=-, \lambda_{N}=-\frac{1}{2}, \lambda_{V}=1\right]}=O_{G_{2}^{-}, r=1}^{\left[J=\frac{5}{2}, P=-, \lambda_{N}=\frac{1}{2}, \lambda_{V}=-1\right]}=O_{G_{2}^{-}, r=1}$

$O_{G_{2}^{-}, r=1}^{\left[J=\frac{5}{2}, L=2, S=\frac{3}{2}\right]}=O_{G_{2}^{-}, r=1}^{\left[J=\frac{5}{2}, L=4, S=\frac{3}{2}\right]}=O_{G_{2}^{-}, r=1}$

$\mathrm{H}^{+}$:

$O_{H^{+}, r=1, n=1}=N_{-\frac{1}{2}}\left(e_{x}\right) V_{x}\left(-e_{x}\right)-N_{-\frac{1}{2}}\left(-e_{x}\right) V_{x}\left(e_{x}\right)-N_{-\frac{1}{2}}\left(e_{y}\right) V_{y}\left(-e_{y}\right)+N_{-\frac{1}{2}}\left(-e_{y}\right) V_{y}\left(e_{y}\right)$ $O_{H^{+}, r=1, n=2}=-N_{-\frac{1}{2}}\left(e_{x}\right) V_{y}\left(-e_{x}\right)+N_{-\frac{1}{2}}\left(-e_{x}\right) V_{y}\left(e_{x}\right)+i N_{\frac{1}{2}}\left(e_{x}\right) V_{z}\left(-e_{x}\right)-i N_{\frac{1}{2}}\left(-e_{x}\right) V_{z}\left(e_{x}\right)$ $-N_{-\frac{1}{2}}\left(e_{y}\right) V_{x}\left(-e_{y}\right)+N_{-\frac{1}{2}}\left(-e_{y}\right) V_{x}\left(e_{y}\right)+N_{\frac{1}{2}}\left(e_{y}\right) V_{z}\left(-e_{y}\right)-N_{\frac{1}{2}}\left(-e_{y}\right) V_{z}\left(e_{y}\right)$ $-2 i N_{\frac{1}{2}}\left(e_{z}\right)\left(V_{x}\left(-e_{z}\right)-i V_{y}\left(-e_{z}\right)\right)+2 N_{\frac{1}{2}}\left(-e_{z}\right)\left(V_{y}\left(e_{z}\right)+i V_{x}\left(e_{z}\right)\right)$

$O_{H^{+}, r=1, n=3}=-N_{-\frac{1}{2}}\left(e_{x}\right) V_{y}\left(-e_{x}\right)+N_{-\frac{1}{2}}\left(-e_{x}\right) V_{y}\left(e_{x}\right)-2 i N_{\frac{1}{2}}\left(e_{x}\right) V_{z}\left(-e_{x}\right)+2 i N_{\frac{1}{2}}\left(-e_{x}\right) V_{z}\left(e_{x}\right)$ $-N_{-\frac{1}{2}}\left(e_{y}\right) V_{x}\left(-e_{y}\right)+N_{-\frac{1}{2}}\left(-e_{y}\right) V_{x}\left(e_{y}\right)-2 N_{\frac{1}{2}}\left(e_{y}\right) V_{z}\left(-e_{y}\right)+2 N_{\frac{1}{2}}\left(-e_{y}\right) V_{z}\left(e_{y}\right)$ $+N_{\frac{1}{2}}\left(e_{z}\right)\left(V_{y}\left(-e_{z}\right)+i V_{x}\left(-e_{z}\right)\right)-i N_{\frac{1}{2}}\left(-e_{z}\right)\left(V_{x}\left(e_{z}\right)-i V_{y}\left(e_{z}\right)\right)$

$O_{H^{+}, r=1}^{\left[J=\frac{3}{2}, P=+, \lambda_{N}= \pm \frac{1}{2}, \lambda_{V}=0\right]}=O_{H^{+}, r=1}^{\left[J=\frac{5}{2}, P=+, \lambda_{N}= \pm \frac{1}{2}, \lambda_{V}=0\right]}=O_{H^{+}, r=1, n=1}$

$O_{H^{+}, r=1}^{\left[J=\frac{3}{2}, P=+, \lambda_{N}=-\frac{1}{2}, \lambda_{V}=1\right]}=O_{H^{+}, r=1}^{\left[J=\frac{3}{2}, P=+, \lambda_{N}=\frac{1}{2}, \lambda_{V}=-1\right]}=$ $=O_{H^{+}, r=1}^{\left[J=\frac{5}{2}, P=+, \lambda_{N}=-\frac{1}{2}, \lambda_{V}=1\right]}=O_{H^{+}, r=1}^{\left[J=\frac{5}{2}, P=+, \lambda_{N}=\frac{1}{2}, \lambda_{V}=-1\right]}=O_{H^{+}, r=1, n=2}$ $O_{H^{+}, r=1}^{\left[J=\frac{3}{2}, P=+, \lambda_{N}=-\frac{1}{2}, \lambda_{V}=-1\right]}=O_{H^{+}, r=1}^{\left[J=\frac{3}{2}, P=+, \lambda_{N}=\frac{1}{2}, \lambda_{V}=1\right]}=$ $=O_{H^{+}, r=1}^{\left[J=\frac{5}{2}, P=+, \lambda_{N}=\frac{1}{2}, \lambda_{V}=1\right]}=O_{H^{+}, r=1}^{\left[J=\frac{5}{2}, P=+, \lambda_{N}=-\frac{1}{2}, \lambda_{V}=-1\right]}=O_{H^{+}, r=1, n=2}+2 O_{H^{+}, r=1, n=3}$ $O_{H^{+}, r=1}^{\left[J=\frac{3}{2}, L=1, S=\frac{3}{2}\right]}=3 O_{H^{+}, r=1, n=1}+i\left(4 O_{H^{+}, r=1, n=2}-O_{H^{+}, r=1, n=3}\right)$ $O_{H^{+}, r=1}^{\left[J=\frac{3}{2}, L=1, S=\frac{1}{2}\right]}=O_{H^{+}, r=1}^{\left[J=\frac{5}{2}, L=3, S=\frac{1}{2}\right]}=3 O_{H^{+}, r=1, n=1}+i\left(O_{H^{+}, r=1, n=2}+2 O_{H^{+}, r=1, n=3}\right)$ $O_{H^{+}, r=1}^{\left[J=\frac{3}{2}, L=3, S=\frac{3}{2}\right]}=O_{H^{+}, r=1}^{\left[J, \frac{5}{2}, L=1, S=\frac{3}{2}\right]}=3 O_{H^{+}, r=1, n=1}-i\left(O_{H^{+}, r=1, n=2}+O_{H^{+}, r=1, n=3}\right)$ $O_{H^{+}, r=1}^{\left[J=\frac{5}{2}, L=3, S=\frac{3}{2}\right]}=12 O_{H^{+}, r=1, n=1}+i\left(O_{H^{+}, r=1, n=2}-4 O_{H^{+}, r=1, n=3}\right)$

$\mathrm{H}^{-}$:

$O_{H^{-}, r=1, n=1}=i N_{\frac{1}{2}}\left(e_{x}\right) V_{x}\left(-e_{x}\right)+i N_{\frac{1}{2}}\left(-e_{x}\right) V_{x}\left(e_{x}\right)+N_{\frac{1}{2}}\left(e_{y}\right) V_{y}\left(-e_{y}\right)+N_{\frac{1}{2}}\left(-e_{y}\right) V_{y}\left(e_{y}\right)$ $O_{H^{-}, r=1, n=2}=-i N_{\frac{1}{2}}\left(e_{x}\right) V_{y}\left(-e_{x}\right)-i N_{\frac{1}{2}}\left(-e_{x}\right) V_{y}\left(e_{x}\right)-N_{-\frac{1}{2}}\left(e_{x}\right) V_{z}\left(-e_{x}\right)-N_{-\frac{1}{2}}\left(-e_{x}\right) V_{z}\left(e_{x}\right)$ $+N_{\frac{1}{2}}\left(e_{y}\right) V_{x}\left(-e_{y}\right)+N_{\frac{1}{2}}\left(-e_{y}\right) V_{x}\left(e_{y}\right)+N_{-\frac{1}{2}}\left(e_{y}\right) V_{z}\left(-e_{y}\right)+N_{-\frac{1}{2}}\left(-e_{y}\right) V_{z}\left(e_{y}\right)$ $+2 N_{\frac{1}{2}}\left(e_{z}\right)\left(V_{x}\left(-e_{z}\right)-i V_{y}\left(-e_{z}\right)\right)+2 N_{\frac{1}{2}}\left(-e_{z}\right)\left(V_{x}\left(e_{z}\right)-i V_{y}\left(e_{z}\right)\right)$

$O_{H^{-}, r=1, n=3}=i N_{\frac{1}{2}}\left(e_{x}\right) V_{y}\left(-e_{x}\right)+i N_{\frac{1}{2}}\left(-e_{x}\right) V_{y}\left(e_{x}\right)-2 N_{-\frac{1}{2}}\left(e_{x}\right) V_{z}\left(-e_{x}\right)-2 N_{-\frac{1}{2}}\left(-e_{x}\right) V_{z}\left(e_{x}\right)$ $-N_{\frac{1}{2}}\left(e_{y}\right) V_{x}\left(-e_{y}\right)-N_{\frac{1}{2}}\left(-e_{y}\right) V_{x}\left(e_{y}\right)+2 N_{-\frac{1}{2}}\left(e_{y}\right) V_{z}\left(-e_{y}\right)+2 N_{-\frac{1}{2}}\left(-e_{y}\right) V_{z}\left(e_{y}\right)$ $+N_{\frac{1}{2}}\left(e_{z}\right)\left(V_{x}\left(-e_{z}\right)-i V_{y}\left(-e_{z}\right)\right)+N_{\frac{1}{2}}\left(-e_{z}\right)\left(V_{x}\left(e_{z}\right)-i V_{y}\left(e_{z}\right)\right)$

$O_{H^{-}, r=1}^{\left[J=\frac{3}{2}, P=-, \lambda_{N}= \pm \frac{1}{2}, \lambda_{V}=0\right]}=O_{H^{-,}, r=1}^{\left[J=\frac{5}{2}, P=-, \lambda_{N}= \pm \frac{1}{2}, \lambda_{V}=0\right]}=O_{H^{-}, r=1, n=1}$ 


$$
\begin{aligned}
& O_{H^{-}, r=1}^{\left[J=\frac{3}{2}, P=-, \lambda_{N}=\frac{1}{2}, \lambda_{V}=-1\right]}=O_{H^{-}, r=1}^{\left[J=\frac{3}{2}, P=-, \lambda_{N}=-\frac{1}{2}, \lambda_{V}=1\right]}= \\
& \quad=O_{H^{-}, r=1}^{\left[J=\frac{5}{2}, P=-, \lambda_{N}=\frac{1}{2}, \lambda_{V}=-1\right]}=O_{H^{-}, r=1}^{\left[J=\frac{5}{2}, P=-, \lambda_{N}=-\frac{1}{2}, \lambda_{V}=1\right]}=O_{H^{-}, r=1, n=2} \\
& \quad=O_{H^{-}, r=1}^{\left[J=\frac{5}{2}, P=-, \lambda_{N}=\frac{1}{2}, \lambda_{V}=1\right]}=O_{H^{-}, r=1}^{\left[J=\frac{5}{2}, P=-, \lambda_{N}=-\frac{1}{2}, \lambda_{V}=-1\right]}=O_{H^{-}, r=1, n=2}-2 O_{H^{-}, r=1, n=3} \\
& O_{\left.H^{-}, r=\frac{3}{2}, P=-, \lambda_{N}=\frac{1}{2}, \lambda_{V}=1\right]}^{[J=1}=O^{\left[J=\frac{3}{2}, P=-, \lambda_{N}=-\frac{1}{2}, \lambda_{V}=-1\right]}= \\
& O_{H^{-}, r=1}^{\left[J=\frac{3}{2}, L=0, S=\frac{3}{2}\right]}=-3 i O_{H^{-}, r=1, n=1}+2 O_{H^{-}, r=1, n=2}-O_{H^{-}, r=1, n=3} \\
& O_{\left.H^{-}, r=\frac{3}{2}, L=2, S=\frac{3}{2}\right]}^{[J=1}=O_{\left.H^{-}, r=\frac{5}{2}, L=2, S=\frac{3}{2}\right]}^{[J=}=3 i O_{H^{-}, r=1, n=1}+O_{H^{-}, r=1, n=2}+O_{H^{-}, r=1, n=3} \\
& O_{H^{-}, r=1}^{\left[J=\frac{3}{2}, L=2, S=\frac{1}{2}\right]}=O_{\left.H^{-}, r=\frac{5}{2}, L=2, S=\frac{1}{2}\right]}^{[J=}=3 i O_{H^{-}, r=1, n=1}+O_{H^{-}, r=1, n=2}-2 O_{H^{-}, r=1, n=3} \\
& O_{H^{-}, r=1}^{\left[J=\frac{5}{2}, L=4, S=\frac{3}{2}\right]}=12 i O_{H^{-}, r=1, n=1}-3 O_{H^{-}, r=1, n=2}+4 O_{H^{-}, r=1, n=3}
\end{aligned}
$$

\section{C.3 NN operators}

\section{C.3.1 $|p|=0$}

$$
\begin{aligned}
& A_{1}^{+}: \\
& O_{A_{1}^{+}, r=1}=\mathrm{N}_{\frac{1}{2}}(0) \mathrm{N}^{\prime}{ }_{-\frac{1}{2}}(0)-\mathrm{N}_{-\frac{1}{2}}(0) \mathrm{N}^{\prime}{ }_{\frac{1}{2}}(0) \\
& O_{A_{1}^{+}, r=1}^{\left[J=0, m_{J}=0, L=0, S=0\right]}=O_{A_{1}^{+}, r=1} \\
& T_{1}^{+}: \\
& O_{T_{1}^{+}, r=1}=\mathrm{N}_{-\frac{1}{2}}(0) \mathrm{N}^{\prime}{ }_{-\frac{1}{2}}(0)-\mathrm{N}_{\frac{1}{2}}(0) \mathrm{N}^{\prime}{ }_{\frac{1}{2}}(0) \\
& O_{T_{1}^{+}, r=1}^{[J=1, L=0, S=1]}=O_{T_{1}^{+}, r=1}
\end{aligned}
$$

Operators for other irreps vanish. For two identical fermions $N=N^{\prime}$, the $O_{T_{1}^{+}}=$ $N_{-\frac{1}{2}}(0) \mathrm{N}_{-\frac{1}{2}}(0)-\mathrm{N}_{\frac{1}{2}}(0) \mathrm{N}_{\frac{1}{2}}(0)=0$ due to anticommutation of nucleon fields. This is also required by the Fermi-Dirac statistics for this case as spin, isospin and spatial parts are all symmetric under exchange of two particles.

\section{C.3.2 $|p|=1$}

$$
O_{\mathrm{A}_{2}^{+}}=O_{\mathrm{A}_{2}^{-}}=0
$$

$\mathrm{A}_{1}^{+}$:

$$
\begin{aligned}
& O_{A_{1}^{+}, r=1}=-\mathrm{N}_{-\frac{1}{2}}\left(e_{x}\right) \mathrm{N}_{\frac{1}{2}}^{\prime}\left(-e_{x}\right)-\mathrm{N}_{-\frac{1}{2}}\left(-e_{x}\right) \mathrm{N}_{\frac{1}{2}}^{\prime}\left(e_{x}\right)+\mathrm{N}_{\frac{1}{2}}\left(e_{x}\right) \mathrm{N}_{-\frac{1}{2}}^{\prime}\left(-e_{x}\right)+\mathrm{N}_{\frac{1}{2}}\left(-e_{x}\right) \mathrm{N}_{-\frac{1}{2}}^{\prime}\left(e_{x}\right) \\
& -\mathrm{N}_{-\frac{1}{2}}\left(e_{y}\right) \mathrm{N}_{\frac{1}{2}}^{\prime}\left(-e_{y}\right)-\mathrm{N}_{-\frac{1}{2}}\left(-e_{y}\right) \mathrm{N}_{\frac{1}{2}}^{\prime}\left(e_{y}\right)+\mathrm{N}_{\frac{1}{2}}\left(e_{y}\right) \mathrm{N}_{-\frac{1}{2}}^{\prime}\left(-e_{y}\right)+\mathrm{N}_{\frac{1}{2}}\left(-e_{y}\right) \mathrm{N}^{\prime}{ }_{-\frac{1}{2}}\left(e_{y}\right) \\
& -\mathrm{N}_{-\frac{1}{2}}\left(e_{z}\right) \mathrm{N}_{\frac{1}{2}}^{\prime}\left(-e_{z}\right)-\mathrm{N}_{-\frac{1}{2}}\left(-e_{z}\right) \mathrm{N}_{\frac{1}{2}}^{\prime}\left(e_{z}\right)+\mathrm{N}_{\frac{1}{2}}\left(e_{z}\right) \mathrm{N}_{-\frac{1}{2}}^{\prime}\left(-e_{z}\right)+\mathrm{N}_{\frac{1}{2}}\left(-e_{z}\right) \mathrm{N}_{-\frac{1}{2}}^{\prime}\left(e_{z}\right) \\
& O_{A_{1}^{+}, r=1}^{\left[J=0, P=+, \lambda_{N}=\frac{1}{2}, \lambda_{N^{\prime}}=\frac{1}{2}\right]}=O_{A_{1}^{+}, r=1}^{\left[J=0, P=+, \lambda_{N}=-\frac{1}{2}, \lambda_{N^{\prime}}=-\frac{1}{2}\right]}=O_{A_{1}^{+}, r=1}^{[J=0, L=0, S=0]}=O_{A_{1}^{+}, r=1} \\
& A_{1}^{-}: \\
& O_{A_{1}^{-}, r=1}:=-\mathrm{N}_{\frac{1}{2}}\left(e_{x}\right) \mathrm{N}_{\frac{1}{2}}^{\prime}\left(-e_{x}\right)+\mathrm{N}_{\frac{1}{2}}\left(-e_{x}\right) \mathrm{N}^{\prime}{ }_{\frac{1}{2}}\left(e_{x}\right)+\mathrm{N}_{-\frac{1}{2}}\left(e_{x}\right) \mathrm{N}^{\prime}{ }_{-\frac{1}{2}}\left(-e_{x}\right)-\mathrm{N}_{-\frac{1}{2}}\left(-e_{x}\right) \mathrm{N}_{-\frac{1}{2}}\left(e_{x}\right) \\
& -i \mathrm{~N}_{\frac{1}{2}}\left(e_{y}\right) \mathrm{N}_{\frac{1}{2}}^{\prime}\left(-e_{y}\right)+i \mathrm{~N}_{\frac{1}{2}}\left(-e_{y}\right) \mathrm{N}_{\frac{1}{2}}^{\prime}\left(e_{y}\right)-i \mathrm{~N}_{-\frac{1}{2}}\left(e_{y}\right) \mathrm{N}_{-\frac{1}{2}}^{\prime}\left(-e_{y}\right)+i \mathrm{~N}_{-\frac{1}{2}}\left(-e_{y}\right) \mathrm{N}_{-\frac{1}{2}}^{\prime}\left(e_{y}\right)
\end{aligned}
$$




$$
\begin{aligned}
& +\mathrm{N}_{-\frac{1}{2}}\left(e_{z}\right) \mathrm{N}_{\frac{1}{2}}^{\prime}\left(-e_{z}\right)-\mathrm{N}_{-\frac{1}{2}}\left(-e_{z}\right) \mathrm{N}_{\frac{1}{2}}^{\prime}\left(e_{z}\right)+\mathrm{N}_{\frac{1}{2}}\left(e_{z}\right) \mathrm{N}_{-\frac{1}{2}}^{\prime}\left(-e_{z}\right)-\mathrm{N}_{\frac{1}{2}}\left(-e_{z}\right) \mathrm{N}_{-\frac{1}{2}}^{\prime}\left(e_{z}\right) \\
& O_{A_{1}^{-}, r=1}^{\left[J=0, P=-, \lambda_{N}=\frac{1}{2}, \lambda_{N^{\prime}}=\frac{1}{2}\right]}=O_{A_{1}^{-}, r=1}^{\left[J=0, P=-, \lambda_{N}=-\frac{1}{2}, \lambda_{N^{\prime}}=-\frac{1}{2}\right]}=O_{A_{1}^{-}, r=1}^{[J=0, L=1, S=1]}=O_{A_{1}^{-}, r=1}
\end{aligned}
$$

$\mathrm{T}_{1}^{+}$:

$$
\begin{aligned}
& O_{T_{1}^{+}, r=1, n=1}=-\mathrm{N}_{\frac{1}{2}}\left(e_{x}\right) \mathrm{N}_{\frac{1}{2}}^{\prime}\left(-e_{x}\right)-\mathrm{N}_{\frac{1}{2}}\left(-e_{x}\right) \mathrm{N}_{\frac{1}{2}}^{\prime}\left(e_{x}\right)+\mathrm{N}_{-\frac{1}{2}}\left(e_{x}\right) \mathrm{N}_{-\frac{1}{2}}^{\prime}\left(-e_{x}\right)+\mathrm{N}_{-\frac{1}{2}}\left(-e_{x}\right) \mathrm{N}_{-\frac{1}{2}}^{\prime}\left(e_{x}\right) \\
& O_{T_{1}^{+}, r=1, n=2}=\left.-\mathrm{N}_{\frac{1}{2}}\left(e_{y}\right) \mathrm{N}_{\frac{1}{2}}^{\prime}\left(-e_{y}\right)-\mathrm{N}_{\frac{1}{2}}\left(-e_{y}\right) \mathrm{N}_{\frac{1}{2}}\left(e_{y}\right)+\mathrm{N}_{-\frac{1}{2}}\left(e_{y}\right) \mathrm{N}_{-\frac{1}{2}}\left(-e_{y}\right)+\mathrm{N}_{-\frac{1}{2}}\left(-e_{y}\right) \mathrm{N}_{-\frac{1}{2}} e_{y}\right) \\
&-\mathrm{N}_{\frac{1}{2}}\left(e_{z}\right) \mathrm{N}_{\frac{1}{2}}\left(-e_{z}\right)-\mathrm{N}_{\frac{1}{2}}\left(-e_{z}\right) \mathrm{N}_{\frac{1}{2}}\left(e_{z}\right)+\mathrm{N}_{-\frac{1}{2}}\left(e_{z}\right) \mathrm{N}_{-\frac{1}{2}}\left(-e_{z}\right)+\mathrm{N}_{-\frac{1}{2}}\left(-e_{z}\right) \mathrm{N}_{-\frac{1}{2}}\left(e_{z}\right) \\
& O_{T_{1}^{+}, r=1}^{\left[J=1, P=+, \lambda_{N}=\frac{1}{2}, \lambda_{N^{\prime}}=\frac{1}{2}\right]}=O_{T_{1}^{+}, r=1}^{\left[J=1, P=+, \lambda_{N}=-\frac{1}{2}, \lambda_{N^{\prime}}=-\frac{1}{2}\right]}=O_{T_{1}^{+}, r=1, n=1} \\
& O_{T_{1}^{+}, r=1}^{\left[J=1, P=+, \lambda_{N}=-\frac{1}{2}, \lambda_{N^{\prime}}=\frac{1}{2}\right]}=O_{T_{1}^{+}, r=1}^{\left[J=1, P=+, \lambda_{N}=\frac{1}{2}, \lambda_{N^{\prime}}=-\frac{1}{2}\right]}=O_{T_{1}^{+}, r=1, n=2} \\
& O_{T_{1}^{+}, r=1}^{[J=1, L=2, S=1]}=2 O_{T_{1}^{+}, r=1, n=1}-O_{T_{1}^{+}, r=1, n=2} \\
& O_{T_{1}^{+}, r=1}^{[J=1, L=0, S=1]}=O_{T_{1}^{+}, r=1, n=1}+O_{T_{1}^{+}, r=1, n=2}
\end{aligned}
$$$$
O_{T_{1}^{+}, r=1, n=2}=-\mathrm{N}_{\frac{1}{2}}\left(e_{y}\right) \mathrm{N}_{\frac{1}{2}}^{\prime}\left(-e_{y}\right)-\mathrm{N}_{\frac{1}{2}}\left(-e_{y}\right) \mathrm{N}_{\frac{1}{2}}^{\prime}\left(e_{y}\right)+\mathrm{N}_{-\frac{1}{2}}\left(e_{y}\right) \mathrm{N}_{-\frac{1}{2}}^{\prime}\left(-e_{y}\right)+\mathrm{N}_{-\frac{1}{2}}\left(-e_{y}\right) \mathrm{N}_{-\frac{1}{2}}^{\prime}\left(e_{y}\right)
$$

$\mathrm{T}_{1}^{-}$:

$O_{T_{1}^{-}, r=1, n=1}=\mathrm{N}_{-\frac{1}{2}}\left(e_{y}\right) \mathrm{N}_{\frac{1}{2}}^{\prime}\left(-e_{y}\right)-\mathrm{N}_{-\frac{1}{2}}\left(-e_{y}\right) \mathrm{N}^{\prime}{ }_{\frac{1}{2}}\left(e_{y}\right)+\mathrm{N}_{\frac{1}{2}}\left(e_{y}\right) \mathrm{N}_{-\frac{1}{2}}\left(-e_{y}\right)-\mathrm{N}_{\frac{1}{2}}\left(-e_{y}\right) \mathrm{N}_{-\frac{1}{2}}\left(e_{y}\right)$ $+i \mathrm{~N}_{\frac{1}{2}}\left(e_{z}\right) \mathrm{N}_{\frac{1}{2}}^{\prime}\left(-e_{z}\right)-i \mathrm{~N}_{\frac{1}{2}}\left(-e_{z}\right) \mathrm{N}_{\frac{1}{2}}^{\prime}\left(e_{z}\right)+i \mathrm{~N}_{-\frac{1}{2}}\left(e_{z}\right) \mathrm{N}^{\prime}{ }_{-\frac{1}{2}}\left(-e_{z}\right)-i \mathrm{~N}_{-\frac{1}{2}}\left(-e_{z}\right) \mathrm{N}_{-\frac{1}{2}}\left(e_{z}\right)$

$O_{T_{1}^{-}, r=1, n=2}=-\mathrm{N}_{-\frac{1}{2}}\left(e_{x}\right) \mathrm{N}^{\prime}{ }_{\frac{1}{2}}\left(-e_{x}\right)+\mathrm{N}_{-\frac{1}{2}}\left(-e_{x}\right) \mathrm{N}^{\prime}{ }_{\frac{1}{2}}\left(e_{x}\right)+\mathrm{N}_{\frac{1}{2}}\left(e_{x}\right) \mathrm{N}^{\prime}{ }_{-\frac{1}{2}}\left(-e_{x}\right)-\mathrm{N}_{\frac{1}{2}}\left(-e_{x}\right) \mathrm{N}^{\prime}{ }_{-\frac{1}{2}}\left(e_{x}\right)$ $O_{T_{1}^{-}, r=1}^{\left[J=1, P=-, \lambda_{N}=\frac{1}{2}, \lambda_{N^{\prime}}=\frac{1}{2}\right]}=O_{T_{1}^{-}, r=1}^{\left[J=1, P=-, \lambda_{N}=-\frac{1}{2}, \lambda_{N^{\prime}}=-\frac{1}{2}\right]}=O_{T_{1}^{-}, r=1, n=2}$ $O_{T_{1}^{-}, r=1}^{\left[J=1, P=-, \lambda_{N}=\frac{1}{2}, \lambda_{N^{\prime}}=-\frac{1}{2}\right]}=O_{T_{1}^{-}, r=1}^{\left[J=1, P=-, \lambda_{N}=-\frac{1}{2}, \lambda_{N^{\prime}}=\frac{1}{2}\right]}=O_{T_{1}^{-}, r=1, n=1}$ $O_{T_{1}^{-}, r=1}^{[J=1, L=1, S=1]}=O_{T_{1}^{-}, r=1, n=1}$ $O_{T_{1}^{-}, r=1}^{[J=1, L=1, S=0]}=O_{T_{1}^{-}, r=1, n=2}$

$\mathrm{T}_{2}^{+}$

$$
\begin{aligned}
O_{T_{2}^{+}, r=1}= & -\mathrm{N}_{\frac{1}{2}}\left(e_{y}\right) \mathrm{N}_{\frac{1}{2}}^{\prime}\left(-e_{y}\right)-\mathrm{N}_{\frac{1}{2}}\left(-e_{y}\right) \mathrm{N}_{\frac{1}{2}}^{\prime}\left(e_{y}\right)+\mathrm{N}_{-\frac{1}{2}}\left(e_{y}\right) \mathrm{N}_{-\frac{1}{2}}^{\prime}\left(-e_{y}\right)+\mathrm{N}_{-\frac{1}{2}}\left(-e_{y}\right) \mathrm{N}_{-\frac{1}{2}}^{\prime}{ }^{\prime}\left(e_{y}\right) \\
& \left.+\mathrm{N}_{\frac{1}{2}}\left(e_{z}\right) \mathrm{N}_{\frac{1}{2}}^{\prime}\left(-e_{z}\right)+\mathrm{N}_{\frac{1}{2}}\left(-e_{z}\right) \mathrm{N}_{\frac{1}{2}}^{\prime}\left(e_{z}\right)-\mathrm{N}_{-\frac{1}{2}}\left(e_{z}\right) \mathrm{N}_{-\frac{1}{2}}\left(-e_{z}\right)-\mathrm{N}_{-\frac{1}{2}}\left(-e_{z}\right) \mathrm{N}_{-\frac{1}{2}} e_{z}\right)
\end{aligned}
$$$$
O_{T_{2}^{+}, r=1}^{\left[J=2, P=+, \lambda_{N}=\frac{1}{2}, \lambda_{N^{\prime}}=-\frac{1}{2}\right]}=O_{T_{2}^{+}, r=1}^{\left[J=2, P=+, \lambda_{N}=-\frac{1}{2}, \lambda_{N^{\prime}}=\frac{1}{2}\right]}=O_{T_{2}^{+}, r=1}^{[J=2, L=2, S=1]}=O_{T_{2}^{+}, r=1}
$$

$\mathrm{T}_{2}^{-}$:

$$
\begin{aligned}
& O_{T_{2}^{-}, r=1}=i \mathrm{~N}_{-\frac{1}{2}}\left(e_{y}\right) \mathrm{N}^{\prime}{ }_{\frac{1}{2}}\left(-e_{y}\right)-i \mathrm{~N}_{-\frac{1}{2}}\left(-e_{y}\right) \mathrm{N}^{\prime}{ }_{\frac{1}{2}}\left(e_{y}\right)+i \mathrm{~N}_{\frac{1}{2}}\left(e_{y}\right) \mathrm{N}_{-\frac{1}{2}}^{\prime}\left(-e_{y}\right)-i \mathrm{~N}_{\frac{1}{2}}\left(-e_{y}\right) \mathrm{N}_{-\frac{1}{2}}^{\prime}\left(e_{y}\right) \\
& +\mathrm{N}_{\frac{1}{2}}\left(e_{z}\right) \mathrm{N}_{\frac{1}{2}}^{\prime}\left(-e_{z}\right)-\mathrm{N}_{\frac{1}{2}}\left(-e_{z}\right) \mathrm{N}_{\frac{1}{2}}^{\prime}\left(e_{z}\right)+\mathrm{N}_{-\frac{1}{2}}\left(e_{z}\right) \mathrm{N}^{\prime}{ }_{-\frac{1}{2}}\left(-e_{z}\right)-\mathrm{N}_{-\frac{1}{2}}\left(-e_{z}\right) \mathrm{N}_{-\frac{1}{2}}^{\prime}\left(e_{z}\right) \\
& O_{T_{2}^{-}, r=1}^{\left[J=2, P=-, \lambda_{N}=\frac{1}{2}, \lambda_{N^{\prime}}=-\frac{1}{2}\right]}=O_{T_{2}^{-}, r=1}^{\left[J=2, P=-, \lambda_{N}=-\frac{1}{2}, \lambda_{N^{\prime}}=\frac{1}{2}\right]}=O_{T_{2}^{-}, r=1}^{[J=2, L=1, S=1]}=O_{T_{2}^{-}, r=1}^{[J=2, L=3, S=1]}=O_{T_{2}^{-}, r=1} \\
& \mathrm{E}^{+}: \\
& O_{E^{+}, r=1}=-\mathrm{N}_{-\frac{1}{2}}\left(e_{x}\right) \mathrm{N}_{\frac{1}{2}}^{\prime}\left(-e_{x}\right)-\mathrm{N}_{-\frac{1}{2}}\left(-e_{x}\right) \mathrm{N}_{\frac{1}{2}}^{\prime}\left(e_{x}\right)+\mathrm{N}_{\frac{1}{2}}\left(e_{x}\right) \mathrm{N}_{-\frac{1}{2}}^{\prime}\left(-e_{x}\right)+\mathrm{N}_{\frac{1}{2}}\left(-e_{x}\right) \mathrm{N}_{-\frac{1}{2}}^{\prime}\left(e_{x}\right) \\
& -\mathrm{N}_{-\frac{1}{2}}\left(e_{y}\right) \mathrm{N}^{\prime}{ }_{\frac{1}{2}}\left(-e_{y}\right)-\mathrm{N}_{-\frac{1}{2}}\left(-e_{y}\right) \mathrm{N}_{\frac{1}{2}}\left(e_{y}\right)+\mathrm{N}_{\frac{1}{2}}\left(e_{y}\right) \mathrm{N}_{-\frac{1}{2}}\left(-e_{y}\right)+\mathrm{N}_{\frac{1}{2}}\left(-e_{y}\right) \mathrm{N}_{-\frac{1}{2}}\left(e_{y}\right) \\
& +2 \mathrm{~N}_{-\frac{1}{2}}\left(e_{z}\right) \mathrm{N}_{\frac{1}{2}}^{\prime}\left(-e_{z}\right)+2 \mathrm{~N}_{-\frac{1}{2}}\left(-e_{z}\right) \mathrm{N}_{\frac{1}{2}}^{\prime}\left(e_{z}\right)-2 \mathrm{~N}_{\frac{1}{2}}\left(e_{z}\right) \mathrm{N}_{-\frac{1}{2}}\left(-e_{z}\right)-2 \mathrm{~N}_{\frac{1}{2}}\left(-e_{z}\right) \mathrm{N}_{-\frac{1}{2}}\left(e_{z}\right) \\
& O_{E^{+}, r=1}^{\left[J=2, P=+, \lambda_{N}=\frac{1}{2}, \lambda_{N^{\prime}}=\frac{1}{2}\right]}=O_{E^{+}, r=1}^{\left[J=2, P=+, \lambda_{N}=-\frac{1}{2}, \lambda_{N^{\prime}}=-\frac{1}{2}\right]}=O_{E^{+}, r=1}^{[J=2, L=2, S=0]}=O_{E^{+}, r=1}
\end{aligned}
$$




$$
\begin{aligned}
& \mathrm{E}^{-}: \\
& O_{E^{-}, r=1}=-\mathrm{N}_{\frac{1}{2}}\left(e_{x}\right) \mathrm{N}^{\prime}{ }_{\frac{1}{2}}\left(-e_{x}\right)+\mathrm{N}_{\frac{1}{2}}\left(-e_{x}\right) \mathrm{N}^{\prime}{ }_{\frac{1}{2}}\left(e_{x}\right)+\mathrm{N}_{-\frac{1}{2}}\left(e_{x}\right) \mathrm{N}^{\prime}{ }_{-\frac{1}{2}}\left(-e_{x}\right)-\mathrm{N}_{-\frac{1}{2}}\left(-e_{x}\right) \mathrm{N}^{\prime}{ }_{-\frac{1}{2}}\left(e_{x}\right) \\
&-i \mathrm{~N}_{\frac{1}{2}}\left(e_{y}\right) \mathrm{N}^{\prime}{ }_{\frac{1}{2}}\left(-e_{y}\right)+i \mathrm{~N}_{\frac{1}{2}}\left(-e_{y}\right) \mathrm{N}^{\prime}{ }_{\frac{1}{2}}\left(e_{y}\right)-i \mathrm{~N}_{-\frac{1}{2}}\left(e_{y}\right) \mathrm{N}^{\prime}{ }_{-\frac{1}{2}}\left(-e_{y}\right)+i \mathrm{~N}_{-\frac{1}{2}}\left(-e_{y}\right) \mathrm{N}^{\prime}{ }_{-\frac{1}{2}}\left(e_{y}\right) \\
&-2 \mathrm{~N}_{-\frac{1}{2}}\left(e_{z}\right) \mathrm{N}_{\frac{1}{2}}^{\prime}\left(-e_{z}\right)+2 \mathrm{~N}_{-\frac{1}{2}}\left(-e_{z}\right) \mathrm{N}_{\frac{1}{2}}^{\prime}\left(e_{z}\right)-2 \mathrm{~N}_{\frac{1}{2}}\left(e_{z}\right) \mathrm{N}^{\prime}{ }_{-\frac{1}{2}}\left(-e_{z}\right)+2 \mathrm{~N}_{\frac{1}{2}}\left(-e_{z}\right) \mathrm{N}^{\prime}{ }_{-\frac{1}{2}}\left(e_{z}\right) \\
& O_{E^{-}, r=1}^{\left[J=2, P=-, \lambda_{N}=\frac{1}{2}, \lambda_{N^{\prime}}=\frac{1}{2}\right]}=O_{E^{-}, r=1}^{\left[J=2, P=-, \lambda_{N}=-\frac{1}{2}, \lambda_{N^{\prime}}=-\frac{1}{2}\right]}=O_{E^{-}, r=1}^{[J=2, L=1, S=1]}=O_{E^{-}, r=1}^{[J=2, L=3, S=1]}=O_{E^{-}, r=1}
\end{aligned}
$$

The $L=0$ and $L=2$ partial waves in the deuteron channel $\left(J^{P}=1^{+}\right.$and $\left.S=1\right)$ could be explored with $O_{T_{1}^{+}}^{[J=1, L=0, S=1]}$ and $O_{T_{1}^{+}}^{[J=1, L=2, S=1]}$, respectively. These handles are expected to be effective since the physical mixing between $(L=0, S=1)$ and $(L=2, S=1)$ is small due to the smallness of the tensor force. ${ }^{18}$

\section{Relation between partial wave and helicity operators}

The proof of the relation (4.13) between partial-wave operators (4.11) and helicity operators (4.8) is presented here. The Clebsch-Gordan coefficient in the partial wave operator (4.11) can be rewritten as

$$
C_{s_{1} m_{s 1}, s_{2} m_{s 2}}^{S m_{S}}=C_{s_{1} m_{s 1}, s_{2} m_{s 2}}^{S m_{S}} \times \delta\left(m_{S}, m_{s 1}+m_{s 2}\right)=C_{s_{1} m_{s 1}, s_{2} m_{s 2}}^{S, m_{s 1}+m_{s 2}}
$$

which leads to

$$
O^{|p|, J, m_{J}, S, L}=\sum_{R \in O}\left(\sum_{m_{L}, m_{S}} C_{L m_{L}, S m_{S}}^{J m_{J}} Y_{L m_{L}}^{*}(\widehat{R p})\right) \sum_{m_{s 1}, m_{s 2}} C_{s_{1} m_{s 1}, m_{2} m_{s 2}}^{S, m_{s 1}+m_{s 2}} H_{m_{s 1}}^{(1)}(R p) H_{m_{s 2}}^{(2)}(-R p) .
$$

Let us first consider the term with the sum over $m_{s 1}$ and $m_{s 2}$. The singlehadron operators $H( \pm R p)$ are expressed in terms of $H\left( \pm p_{z}\right)$ via $H_{m_{s}}\left(R^{\prime} p_{z}\right)=$ $\sum_{\lambda} D_{m_{s} \lambda}\left(R^{\prime}\right) R^{\prime} H_{\lambda}\left(p_{z}\right)\left(R^{\prime}\right)^{-1}(2.2)^{19}$ with $R p=R R_{0}^{p} p_{z}=R^{\prime} p_{z}$. Then products of two Wigner D-matrices are reduced via (A.5) and subsequently the product of two CG's via (A.6) leading to

$$
\begin{aligned}
& \sum_{m_{s 1}, m_{s 2}} C_{s_{1} m_{s 1}, s_{2} m_{s 2}}^{S, m_{s 1}+m_{s 2}} H_{m_{s 1}}^{(1)}(R p) H_{m_{s 2}}^{(2)}(-R p)= \\
& =\sum_{m_{s 1}, m_{s 2}} C_{s_{1} m_{s 1}, s_{2} m_{s 2}}^{S, m_{s 1}+m_{s 2}} \sum_{\lambda_{1}, \lambda_{2}} D_{m_{s 1}, \lambda_{1}}^{s_{1}}\left(R R_{0}^{p}\right) D_{m_{s 2},-\lambda_{2}}^{s_{2}}\left(R R_{0}^{p}\right)\left(R R_{0}^{p}\right) H_{\lambda_{1}}^{(1)}\left(p_{z}\right) H_{-\lambda_{2}}^{(2)}\left(-p_{z}\right)\left(R R_{0}^{p}\right)^{-1} \\
& =\sum_{m_{s 1}, m_{s 2}, \lambda_{1}, \lambda_{2}} \sum_{\lambda, m_{S}^{\prime}} \sum_{\tilde{S}} C_{s_{1} m_{s 1}, s_{2} m_{s 2}}^{S, m_{s 1}+m_{s 2}} C_{s_{1} m_{s 1}, \tilde{S}_{2}, m_{s}^{\prime}}^{\prime} m_{s 2} C_{s_{1} \lambda_{1}, s_{2}-\lambda_{2}}^{\tilde{S}, \lambda} D_{m_{S}^{\prime}, \lambda}^{\tilde{S}}\left(R R_{0}^{p}\right) \\
& \quad \times\left(R R_{0}^{p}\right) H_{\lambda_{1}}^{(1)}\left(p_{z}\right) H_{-\lambda_{2}}^{(2)}\left(-p_{z}\right)\left(R R_{0}^{p}\right)^{-1} \\
& =\sum_{m_{S}^{\prime}, \lambda_{1}, \lambda_{2}, \lambda} C_{s_{1} \lambda_{1}, s_{2}-\lambda_{2}}^{S, \lambda} D_{m_{S}^{\prime}, \lambda}^{S}\left(R R_{0}^{p}\right)\left(R R_{0}^{p}\right) H_{\lambda_{1}}^{(1)}\left(p_{z}\right) H_{-\lambda_{2}}^{(2)}\left(-p_{z}\right)\left(R R_{0}^{p}\right)^{-1} .
\end{aligned}
$$

\footnotetext{
${ }^{18}$ The prospects for the lattice extraction of this mixing has been analytically explored in [27].

${ }^{19}$ The summation index is denoted $-\lambda_{2}$ for the second hadron.
} 
The first part of (D.1) contains the spherical harmonics, which is expressed in terms of the Wigner-D functions as

$$
\begin{gathered}
\sum_{m_{L}, m_{S}} C_{L m_{L}, S m_{S}}^{J m_{J}} Y_{L m_{L}}^{*}(\widehat{R p})=\sum_{m_{L}, m_{S}} C_{L m_{L}, S m_{S}}^{J m_{J}} Y_{L m_{L}}^{*}\left(\widehat{R R_{0}^{p} p_{z}}\right)= \\
=\sqrt{\frac{2 L+1}{4 \pi}} \sum_{\lambda=-S}^{S} \sum_{m_{S}} C_{L 0, S \lambda}^{J \lambda}\left(D_{m_{S}, \lambda}^{S}\left(R R_{0}^{p}\right)\right)^{*} D_{m_{J}, \lambda}^{J}\left(R R_{0}^{p}\right) .
\end{gathered}
$$

which will be derived at the end of this appendix.

The partial wave operator now reads

$$
\begin{aligned}
& O^{|p|, J, m_{J}, S, L}=\sqrt{\frac{2 L+1}{4 \pi}} \sum_{\lambda=-S}^{S} \sum_{\lambda_{1}, \lambda_{2}} \sum_{R \in O^{(2)}} \sum_{m_{S}, m_{S}^{\prime}} C_{s_{1} \lambda_{1}, s_{2}-\lambda_{2}}^{S \lambda} C_{L 0, S \lambda}^{J \lambda} \\
& \times D_{m_{S}^{\prime}, \lambda}^{S}\left(R R_{0}^{p}\right)\left(D_{m_{S}, \lambda}^{S}\left(R R_{0}^{p}\right)\right)^{*} D_{m_{J}, \lambda}^{J}\left(R R_{0}^{p}\right)\left(R R_{0}^{p}\right) H_{\lambda_{1}}^{(1)}\left(p_{z}\right) H_{-\lambda_{2}}^{(2)}\left(-p_{z}\right)\left(R R_{0}^{p}\right)^{-1}
\end{aligned}
$$

which can be further simplified with use of Wigner-D function properties ${ }^{20}$

$$
\begin{aligned}
O^{|p|, J, m_{J}, S, L}= & \sqrt{\frac{2 L+1}{4 \pi}} \sum_{\lambda=-S}^{S} \sum_{\lambda_{1}, \lambda_{2}} C_{s_{1} \lambda_{1}, s_{2}-\lambda_{2}}^{S \lambda} C_{L 0, S \lambda}^{J \lambda} \\
& \times \sum_{\lambda^{\prime}} D_{\lambda^{\prime}, \lambda}^{J}\left(R_{0}^{p}\right) \sum_{R \in O^{(2)}} D_{m_{J}, \lambda^{\prime}}^{J}(R)\left(R R_{0}^{p}\right) H_{\lambda_{1}}^{(1)}\left(p_{z}\right) H_{-\lambda_{2}}^{(2)}\left(-p_{z}\right)\left(R R_{0}^{p}\right)^{-1}
\end{aligned}
$$

This operator already has a good parity $P=P_{1} P_{2}(-1)^{L}$ and one can act on it by the parity projection $\frac{1}{2}(O+P I O I)$ without modifying it,

$$
\begin{aligned}
& O^{|p|, J, m_{J}, S, L}=\sqrt{\frac{2 L+1}{4 \pi}} \sum_{\lambda=-S}^{S} \sum_{\lambda_{1}, \lambda_{2}} \sum_{\lambda^{\prime}} D_{\lambda^{\prime}, \lambda}^{J}\left(R_{0}^{p}\right) C_{s_{1} \lambda_{1}, s_{2}-\lambda_{2}}^{S \lambda} C_{L 0, S \lambda}^{J \lambda} \\
& \times \frac{1}{2} \sum_{R \in O^{(2)}} D_{m_{J}, \lambda^{\prime}}^{J}(R)\left(R R_{0}^{p}\right)\left(H_{\lambda_{1}}^{(1)}\left(p_{z}\right) H_{-\lambda_{2}}^{(2)}\left(-p_{z}\right)+P I H_{\lambda_{1}}^{(1)}\left(p_{z}\right) H_{-\lambda_{2}}^{(2)}\left(-p_{z}\right) I\right)\left(R R_{0}^{p}\right)^{-1} .
\end{aligned}
$$

The second line represents the helicity operator (4.8), so the partial wave operator is the following linear combination of helicity operators:

$$
O^{|p|, J, m_{J}, S, L}=\sqrt{\frac{2 L+1}{4 \pi}} \sum_{\lambda=-S}^{S} \sum_{\lambda_{1}, \lambda_{2}} \sum_{\lambda^{\prime}} D_{\lambda^{\prime}, \lambda}^{J}\left(R_{0}^{p}\right) C_{L 0, S \lambda}^{J \lambda} C_{s_{1} \lambda_{1}, s_{2}-\lambda_{2}}^{S \lambda} O^{|p|, J, m_{J}, \lambda^{\prime}, \lambda_{1}, \lambda_{2}} .
$$

A similar relation can be found in eq. (4) of [11].

We close by deriving the relation (D.3), which was used above. The intermediate steps are taken from [28]. Relation $Y_{J m_{J}}^{L S}(\widehat{R p})=\sum_{m_{L}, m_{S}} C_{L m_{L}, S m_{S}}^{J m_{L}} Y_{L m_{L}}(\widehat{R p}) \chi_{S m_{S}}$ (page 196 of [28]) renders via $\chi_{S, m_{S}^{\prime}}^{\dagger} \chi_{S, m_{S}}=\delta_{m_{s} m_{s}^{\prime}}$ the desired product

$$
\begin{gathered}
\sum_{m_{L}, m_{S}} C_{L m_{L}, S m_{S}}^{J m_{J}} Y_{L m_{L}}^{*}(\widehat{R p})=\sum_{m_{S}}\left(\chi_{S m_{S}}^{\dagger} Y_{J m_{J}}^{L S}(\widehat{R p})\right)^{\dagger} . \\
{ }^{20} \sum_{\lambda} D_{m_{S}^{\prime}, \lambda}^{S}\left(R R_{0}^{p}\right)\left(D_{m_{S}, \lambda}^{S}\left(R R_{0}^{p}\right)\right)^{*}=\delta_{m_{S}, m_{S}^{\prime}} \text { and } D_{m_{J}, \lambda}^{J}\left(R R_{0}^{p}\right)=\sum_{\lambda^{\prime}} D_{m_{J}, \lambda^{\prime}}^{J}(R) D_{\lambda^{\prime}, \lambda}^{J}\left(R_{0}^{p}\right) .
\end{gathered}
$$


The tensor $Y_{J m_{J}}^{L S}(\widehat{R p})$ can be transformed to helicity basis (denoted by prime on page 197 of [28]) which in turn can be expressed using Wigner-D matrices (page 197 of [28])

$$
Y_{J m_{J}}^{L S}(\theta, \phi)=\sum_{\lambda=-S}^{S}\left[Y_{J m_{J}}^{L S}(\theta, \phi)\right]^{\prime \lambda} \chi_{S \lambda}^{h}(\theta, \phi)
$$

where the right-hand-side can be further expressed as (pages 173 and 197 of [28])

$$
\left[Y_{J m_{J}}^{L S}(\theta, \phi)\right]^{\prime \lambda}=\sqrt{\frac{2 L+1}{4 \pi}} C_{L 0, S \lambda}^{J \lambda} D_{-\lambda-m_{J}}^{J}(0, \theta, \phi), \quad \chi_{S \lambda}^{h}(\theta, \phi)=\sum_{m_{S}^{\prime}} D_{m_{S}^{\prime} \lambda}^{S}(\phi, \vartheta, 0) \chi_{S m_{S}^{\prime}}
$$

Inserting all to (D.8) and taking into account $D_{-\lambda-m_{J}}^{J}(\alpha, \beta, \gamma)=D_{m_{J} \lambda}^{J}(\gamma, \beta, \alpha)$ one arrives at

$$
\sum_{m_{L}, m_{S}} C_{L m_{L}, S m_{S}}^{J m_{J}} Y_{L m_{L}}^{*}(\theta, \phi)=\sqrt{\frac{2 L+1}{4 \pi}} \sum_{\lambda=-S}^{S} \sum_{m_{S}} C_{L 0, S \lambda}^{J \lambda}\left(D_{m_{S}, \lambda}^{S}(\phi, \theta, 0)\right)^{*} D_{m_{J}, \lambda}^{J}(\phi, \theta, 0) .
$$

This is equivalent to (D.3) for polar angles $\theta=\cos ^{-1}\left[(\widehat{R p})_{z} /|\widehat{R p}|\right]$ and $\phi=-i \log \left[\left((\widehat{R p})_{x}+\right.\right.$ $\left.\left.i(\widehat{R p})_{y}\right) / \sqrt{(\widehat{R p})_{x}^{2}+(\widehat{R p})_{y}^{2}}\right]$ of $R p=R R_{0}^{p} p_{z}$. The rotation of $p_{z}$ with Euler angles $(\phi, \theta, 0)$ leads to the vector with polar coordinates $(\theta, \phi)$.

Open Access. This article is distributed under the terms of the Creative Commons Attribution License (CC-BY 4.0), which permits any use, distribution and reproduction in any medium, provided the original author(s) and source are credited.

\section{References}

[1] E. Berkowitz et al., Two-nucleon higher partial-wave scattering from lattice QCD, Phys. Lett. B 765 (2017) 285 [arXiv:1508.00886] [INSPIRE].

[2] M. Lüscher, Two particle states on a torus and their relation to the scattering matrix, Nucl. Phys. B 354 (1991) 531 [inSPIRE].

[3] R.A. Briceno, Two-particle multichannel systems in a finite volume with arbitrary spin, Phys. Rev. D 89 (2014) 074507 [arXiv:1401.3312] [INSPIRE].

[4] T. Luu and M.J. Savage, Extracting scattering phase-shifts in higher partial-waves from lattice QCD calculations, Phys. Rev. D 83 (2011) 114508 [arXiv:1101.3347] [INSPIRE].

[5] M. Göckeler et al., Scattering phases for meson and baryon resonances on general moving-frame lattices, Phys. Rev. D 86 (2012) 094513 [arXiv:1206.4141] [INSPIRE].

[6] V. Bernard, D. Hoja, U.-G. Meißner and A. Rusetsky, Matrix elements of unstable states, JHEP 09 (2012) 023 [arXiv: 1205.4642] [INSPIRE].

[7] R.A. Briceño, Z. Davoudi and T.C. Luu, Two-nucleon systems in a finite volume: (I) quantization conditions, Phys. Rev. D 88 (2013) 034502 [arXiv:1305.4903] [INSPIRE].

[8] J.J. Dudek, R.G. Edwards, M.J. Peardon, D.G. Richards and C.E. Thomas, Toward the excited meson spectrum of dynamical QCD, Phys. Rev. D 82 (2010) 034508 [arXiv: 1004.4930] [INSPIRE]. 
[9] S.J. Wallace, Partial-wave and helicity operators for the scattering of two hadrons in lattice QCD, Phys. Rev. D 92 (2015) 034520 [arXiv:1506.05492] [INSPIRE].

[10] C.E. Thomas, R.G. Edwards and J.J. Dudek, Helicity operators for mesons in flight on the lattice, Phys. Rev. D 85 (2012) 014507 [Erratum ibid. D 85 (2012) 039901] [arXiv:1107.1930] [INSPIRE].

[11] J.J. Dudek, R.G. Edwards and C.E. Thomas, $S$ and D-wave phase shifts in isospin-2 $\pi \pi$ scattering from lattice QCD, Phys. Rev. D 86 (2012) 034031 [arXiv:1203.6041] [INSPIRE].

[12] D.C. Moore and G.T. Fleming, Multiparticle states and the hadron spectrum on the lattice, Phys. Rev. D 74 (2006) 054504 [hep-lat/0607004] [inSPIRE].

[13] M. Jacob and G.C. Wick, On the general theory of collisions for particles with spin, Annals Phys. 7 (1959) 404 [Annals Phys. 281 (2000) 774] [INSPIRE].

[14] Particle Data Group collaboration, K.A. Olive et al., Review of particle physics, Chin. Phys. C 38 (2014) 090001 [InSPIRE].

[15] S. Weinberg, The quantum theory of fields I, Cambridge University Press, Cambridge U.K. (1995) [INSPIRE].

[16] V. Bernard, M. Lage, U.-G. Meißner and A. Rusetsky, Resonance properties from the finite-volume energy spectrum, JHEP 08 (2008) 024 [arXiv:0806.4495] [INSPIRE].

[17] D.J. Wilson, J.J. Dudek, R.G. Edwards and C.E. Thomas, Resonances in coupled $\pi K, \eta K$ scattering from lattice QCD, Phys. Rev. D 91 (2015) 054008 [arXiv:1411.2004] [inSPIRE].

[18] G. Moir, M. Peardon, S.M. Ryan, C.E. Thomas and D.J. Wilson, Coupled-channel D $\pi, D \eta$ and $D_{s} \bar{K}$ scattering from lattice QCD, JHEP 10 (2016) 011 [arXiv:1607.07093] [INSPIRE].

[19] J. Elliot and P. Dawber, Symmetry in physics I, Oxford University Press, Oxford U.K. (1979).

[20] R.G. Edwards, J.J. Dudek, D.G. Richards and S.J. Wallace, Excited state baryon spectroscopy from lattice QCD, Phys. Rev. D 84 (2011) 074508 [arXiv:1104.5152] [INSPIRE].

[21] N. Isgur and M.B. Wise, Spectroscopy with heavy quark symmetry, Phys. Rev. Lett. 66 (1991) 1130 [INSPIRE].

[22] D. Mohler, S. Prelovsek and R.M. Woloshyn, D $\pi$ scattering and $D$ meson resonances from lattice QCD, Phys. Rev. D 87 (2013) 034501 [arXiv: 1208.4059] [INSPIRE].

[23] E. Wigner, Group theory, Academic Press, Cambridge U.S.A. (1959).

[24] Wolfram Research Inc., Mathematica version 10.4, Champaign U.S.A. (2016).

[25] Lattice Hadron Physics (LHPC) collaboration, S. Basak et al., Clebsch-Gordan construction of lattice interpolating fields for excited baryons, Phys. Rev. D 72 (2005) 074501 [hep-lat/0508018] [INSPIRE].

[26] C.B. Lang, L. Leskovec, M. Padmanath and S. Prelovsek, Pion-nucleon scattering in the Roper channel from lattice $Q C D$, Phys. Rev. D in print [arXiv:1610.01422] [INSPIRE].

[27] R.A. Briceño, Z. Davoudi, T. Luu and M.J. Savage, Two-nucleon systems in a finite volume. II. ${ }^{3} S_{1}-{ }^{3} D_{1}$ coupled channels and the deuteron, Phys. Rev. D 88 (2013) 114507 [arXiv: 1309.3556] [INSPIRE].

[28] D. Varshalovich, Quantum theory of angular momentum, World Scientific, Singapore (1988) [INSPIRE]. 